\title{
A Digital Preclinical PET/MRI Insert and Initial Results
}

\author{
Bjoern Weissler*, Pierre Gebhardt, Peter M. Dueppenbecker, Jakob Wehner, David Schug, \\ Christoph W. Lerche, Benjamin Goldschmidt, Andre Salomon, Iris Verel, Edwin Heijman, \\ Michael Perkuhn, Dirk Heberling, Rene M. Botnar, Fabian Kiessling, and Volkmar Schulz
}

\begin{abstract}
Combining Positron Emission Tomography (PET) with Magnetic Resonance Imaging (MRI) results in a promising hybrid molecular imaging modality as it unifies the high sensitivity of PET for molecular and cellular processes with the functional and anatomical information from MRI. Digital Silicon Photomultipliers (dSiPMs) are the digital evolution in scintillation light detector technology and promise high PET SNR. DSiPMs from Philips Digital Photon Counting (PDPC) were used to develop a preclinical PET/RF gantry with 1-mm scintillation crystal pitch as an insert for clinical MRI scanners. With three exchangeable RF coils, the hybrid field of view has a maximum size of $160 \mathrm{~mm} \times 96.6$ $\mathrm{mm}$ (transaxial $\times$ axial). $\quad 0.1 \mathrm{ppm}$ volume-root-mean-square $B_{0}$-homogeneity is kept within a spherical diameter of $96 \mathrm{~mm}$ (automatic volume shimming). Depending on the coil, MRI SNR
\end{abstract}

Manuscript received March 03, 2015; revised April 21, 2015; accepted April 24, 2015. Date of publication April 29, 2015; date of current version October 28,2015 . This work was co-funded by the German federal state North Rhine Westphalia (HighTech.NRW) and the European Union (European Regional Development Fund: Investing In Your Future) in the ForSaTum project (Grant z0903ht014g); the Centre of Excellence in Medical Engineering funded by the Wellcome Trust and EPSRC under Grant WT 088641/Z/09/Z; and the EU FP7 project SUBLIMA, Grant agreement No 241711. Asterisk indicates corresponding author

This paper has supplementary downloadable material available at http://ieeexplore.ieee.org, provided by the authors. A PDF document contains all supplementary figures and tables, and a movie clip shows the dual-gated PET/MRI CINE images of the mouse heard.

*B. Weissler is with the Clinical Application Research Department of Philips Research, 52074 Aachen, Germany, and with the Department of Physics of Molecular Imaging Systems, Institute of Experimental Molecular Imaging, RWTH Aachen University, 52074 Aachen, Germany (e-mail: bjoern.weissler@philips.com).

P. M. Dueppenbecker and B. Goldschmidt were with the Department of Physics of Molecular Imaging Systems, Institute of Experimental Molecular Imaging, RWTH Aachen University, 52074 Aachen, Germany.

P. Gebhardt and R. M. Botnar are with the Division of Imaging Sciences and Biomedical Engineering, King's College London, WC2R 2LS London, U.K.

J. Wehner, and D. Schug are with the Department of Physics of Molecular Imaging Systems, Institute of Experimental Molecular Imaging, RWTH Aachen University, 52074 Aachen, Germany.

A. Salomon, I. Verel, and E. Heijman are with the Oncology Solutions Department of Philips Research, 5656 AE Eindhoven, The Netherlands.

C. W. Lerche was with the Oncology Solutions Department of Philips Research, 5656 AE Eindhoven, The Netherlands.

M. Perkuhn is with the Clinical Application Research Department of Philips Research, 52074 Aachen, Germany.

D. Heberling is with the Institute of High Frequency Technology, RWTH Aachen University, 52074 Aachen, Germany.

F. Kiessling is with the Institute of Experimental Molecular Imaging, RWTH Aachen University, 52074 Aachen, Germany.

V. Schulz is with the Department of Physics of Molecular Imaging Systems, Institute of Experimental Molecular Imaging, RWTH Aachen University, 52074 Aachen, Germany, and with the Clinical Application Research Department of Philips Research, 52074 Aachen, Germany (e-mail: schulz@pmi.rwth-aachen. de).

Color versions of one or more of the figures in this paper are available online at http://ieeexplore.ieee.org.

Digital Object Identifier 10.1109/TMI.2015.2427993 is decreased by $13 \%$ or $5 \%$ by the PET system. PET count rates, energy resolution of $12.6 \%$ FWHM, and spatial resolution of 0.73 $\mathrm{mm}^{3}$ (isometric volume resolution at isocenter) are not affected by applied MRI sequences. PET time resolution of 565 ps (FWHM) degraded by 6 ps during an EPI sequence. Timing-optimized settings yielded 260 ps time resolution. PET and MR images of a hot-rod phantom show no visible differences when the other modality was in operation and both resolve $0.8-\mathrm{mm}$ rods. Versatility of the insert is shown by successfully combining multi-nuclei MRI $\left({ }^{1} \mathrm{H} /{ }^{19} \mathrm{~F}\right)$ with simultaneously measured PET $\left({ }^{18} \mathrm{~F}\right.$-FDG). A longitudinal study of a tumor-bearing mouse verifies the operability, stability, and in vivo capabilities of the system. Cardiacand respiratory-gated PET/MRI motion-capturing (CINE) images of the mouse heart demonstrate the advantage of simultaneous acquisition for temporal and spatial image registration.

Index Terms-Magnetic resonance imaging (MRI), molecular imaging, PET/MRI, positron emission tomography (PET).

\section{INTRODUCTION}

I $\mathrm{N}$ modern medicine and research, molecular, functional and anatomical information from different imaging modalities are combined to improve diagnostic accuracy [1]. With the introduction of Positron Emission Tomography/Computed Tomography (PET/CT) [2] first hybrid imaging modality was implemented in the clinical routine, which improved registration accuracy and workflow. The combination of PET with Magnetic Resonance Imaging (MRI) reveals comprehensive and complementary information [3] and thus promises to be a valuable tool in research [4]. Multiple clinical applications are already suggested [5], often restricted to simultaneous PET/MRI [6].

Combining the two modalities into a single system introduces several technological challenges [7]. E.g., photomultiplier tubes (PMTs), important elements of standard PET detectors, need to keep a certain distance from the MRI bore and require magnetic shielding. As such, sequential PET/MR scanner can be realized, which are commercially available as clinical [8] and preclinical [9] systems.

The first approach for simultaneous PET/MR used optical fibers to transport light from the scintillating crystals to PMTs outside the MRI [10]. The operation of PET detectors inside the MRI bore became possible by replacing PMTs with solid state detectors [11]. Avalanche Photo Diodes (APDs) were built into several preclinical research inserts [12] and are used in the only simultaneous PET/MRI system being currently commercially available [13]. A detailed history can be found in [7].

A further development of APDs are silicon photomultiplier (SiPM), which have a higher gain and a faster response time than 
APDs. Therefore, these can be employed to measure Time-OfFlight (TOF) information, which increases the PET image SNR [14]. SiPMs were used to build the first PET/MR inserts [15]. Often, long cables were used to connect the detectors inside the MRI bore to the readout electronics [16], but long cables are a potential source of signal degradation and interaction with the MRI scanner [17]. Therefore, our approach (first presented in [15]) is to move the digitization as close as possible to the sensors, and thus into the PET detector module. This concept is also used in the next simultaneous clinical PET/MRI system, which will probably be available soon (FDA approval is currently pending, first systems are already used in research) [18]. On the other hand, this approach places the entire PET detector electronics into a harsh electromagnetic environment: although the regions of possible interaction may be smaller, the fields are much stronger. Furthermore, electronic devices inside the MR bore have a high risk of influencing the MRI scanner: the homogeneity of the static magnetic field $\left(\mathrm{B}_{0}\right)$ is distorted by electronic components [19], the gradient fields are influenced by eddy currents induced in conductive areas [20], and the RF system receives spurious signals from switching currents [21]. To investigate and mitigate these effects, we have built a preclinical PET system with digitization in the PET detector module as an insert for clinical 3-T MRI scanner [22]. The result was that integrated digitization was possible, but that the design needed further improvements.

A next evolutionary step in solid state light sensor design was introduced by Philips in 2009 [23]: Digital Silicon Photomultipliers (dSiPMs), also known as Digital Photon Counters (DPCs). These integrate the time-of-arrival measurement and the counting of SiPM-cell breakdowns directly into the sensor silicon. For PET, this promises excellent time- and energy resolution [24]. And as vulnerable analog signal transmission lines are completely eliminated, DPCs are a good candidate for PET/MRI integration.

\section{A. Aim and Structure}

Encouraged by the results of our PET/RF insert with integrated digitization [22], we decided to integrate this new sensor technology and to improve the complete system in the same step. The resulting design concept of the world's first preclinical PET/MRI insert using fully digital silicon photomultiplier technology was presented in [25]. To our knowledge, this is also the first preclinical dSiPM-based PET system. The aim of this paper is to introduce the completed PET/MRI insert "Hyperion $\mathrm{II}^{\mathrm{D}}$ ", to detail the technical improvements, and to demonstrate their effectiveness in experiments.

Firstly, the key results and identified issues of the previous insert are discussed. Then, the new insert is presented with a focus on the improvements made. Finally, PET/MRI compatibility-, phantom- and first in vivo studies are performed to evaluate the presented changes. When applicable, the measurements are (with appropriate adaptations) performed similar to [22] to allow a comparison between the inserts. A more detailed assessment of the MRI compatibility can be found in [26].

\section{B. Previous Insert "Hyperion I"}

The previous insert, as presented in [22], was only populated with one PET detector ring, resulting in an axial PET Field Of
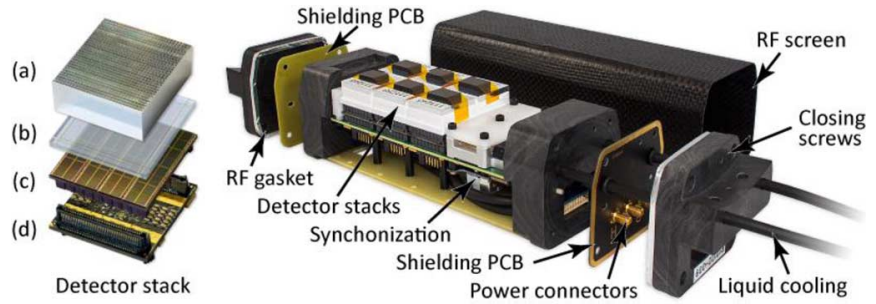

Fig. 1. DPC-based detector stack (left) with scintillation crystal array (a), light guide (b), sensor- (c), and interface board (d). An SDM (right) contains up to six detector stacks. The carbon fiber shield is pushed over the module and the $\mathrm{RF}$ screen is closed on both sides by shielding plates.

View (FOV) of $30.1 \mathrm{~mm}$. Having an energy resolution of $29.7 \%$ and a time resolution of $2.5 \mathrm{~ns}$, it achieved a spatial resolution of $1.3 \mathrm{~mm}$ and a peak sensitivity of $0.6 \%$ [27]. $\mathrm{B}_{0}$-distortion was kept below $2 \mathrm{ppm}$ peak-to-peak in a diameter of $56 \mathrm{~mm}$ within the hybrid FOV. However, $0.1 \mathrm{ppm}$ Volume-Root-Mean-Square (VRMS) homogeneity, important for advanced MR sequences such as spectroscopy, could (even with shimming) only be kept within a diameter of $8 \mathrm{~mm}$. Spurious signal scans revealed RF noise originating from the PET electronics, which presented as dotted lines ("zipper artifacts") in some of the MR images. Further experiments indicated that these signals leaked out of connections between the PET detector modules and the synchronization cables. During long EPI sequences, induced eddy currents resulted in heating of the PET electronics and consequently in a slight adjustment of its count rates (influencing sensitivity and quantification). Additionally, the eddy currents presented as ghosting effects in the MR images: 48 of the 184 taken EPI images showed ghosting, in 14 images ghosting was severe. As a first prototype, the old insert had furthermore a few deficits hindering advanced in vivo imaging: The system was complicated to set up, the integrated MRI RF coil was not exchangeable (often resulting in suboptimal MRI SNR), and it did not have inputs for external trigger signals. All these issues are addressed in the design of the new insert, and the improvements are detailed in the following chapter.

\section{PET/MRI INSERT "HYPERION II ${ }^{\mathrm{D}}$ "}

\section{A. Singles Detection Module (SDM)}

The SDM (Fig. 1) is organized in detector stacks containing four layers: scintillation crystal array, light guide, sensor board, and interface board. The scintillation arrays are made from 30 $\times 3012 \mathrm{~mm}$ long cerium-doped lutetium yttrium orthosilicate (LYSO) crystals (Agile, Knoxville, USA); optically isolated by $67 \mu \mathrm{m}$ Vikuity ${ }^{\mathrm{TM}}$ ESR films (3M, St. Paul, USA). The crystal pitch is $1 \mathrm{~mm}$. Scintillation light is coupled to the sensor board through a borosilicate glass light guide (2-mm thick with $1.3-\mathrm{mm}$ deep slits, filled with white paint, to partly isolate the outer crystals). Optical interfacing is achieved with a dual-component silicon glue (Scionix, Utrecht, the Netherlands). The custom-made sensor board uses $4 \times 4$ DPC dies DLS 3200-22 (Philips Digital Photon Counting, Aachen, Germany), each having $2 \times 2$ DPC sensor channels (pixel). The sensor board is electrically split into two sides to avoid conductive loops via the two connectors. 
TABLE I

Details of THE Detector StACK

\begin{tabular}{l|l}
\hline \hline Parameter & Value \\
\hline Scintillation crystal pitch / size & $1 \mathrm{~mm} / 0.933 \times 0.933 \times 12 \mathrm{~mm}^{3}$ \\
Sensor type / Microcells per channel & PDPC DLS 3200-22/3200 \\
DPC sensor channel (pixel) pitch / size & $4 \mathrm{~mm} / 3.9 \times 3.2 \mathrm{~mm}^{2}$ \\
\hline \hline
\end{tabular}

Data from the DPCs are read out and are pre-processed by a Field Programmable Gate Array (FPGA) (Spartan-6, Xilinx, San Jose, USA) on the interface board. The bias voltage for the DPCs is regulated on the same Printed Circuit Board (PCB). Detailed numbers and dimensions of the detector stack are displayed in Table I and Suppl. fig. 1. A comprehensive introduction to the detector stack is given in [28].

The mainboard of the SDM, the Singles Processing Unit (SPU) [29], supplies and controls $3 \times 2$ stacks. An FPGA (Xilinx Virtex-5) processes the data from the detector stacks [30] and sends them to the Data Acquisition and Processing Server (DAPS). To improve $\mathrm{B}_{0}$-homogeneity, the laser transceivers for optical glass fiber communication (used in the previous insert) were replaced by transceivers for Plastic Optical Fibers (POF), developed as a non-magnetic version for this project [29]. The magnetic material in the SDM was further reduced by replacing more components with carefully selected, partly especially manufactured, versions. Also most semi-manufactured material was inspected prior to processing, since magnetic properties are not normally specified and the exact combination of alloys and additives may vary in different batches.

An SDM has an approximate power dissipation of $30 \mathrm{~W}$ and is cooled with a combined liquid $\left(-5^{\circ} \mathrm{C}\right.$ to $\left.15^{\circ} \mathrm{C}\right)$ and dry air (here: $14^{\circ} \mathrm{C}$, dew point $-21^{\circ} \mathrm{C}$ ) chilling system. The dry air leaving the SDMs is guided by covers through the complete insert. This reduces condensation problems and thus allows lower liquid cooling temperatures compared to the old insert. A second improvement is the direct cooling of the sensor boards and bias voltage regulation circuits (see Suppl. fig. 2), to stabilize their temperature during long MRI EPI sequences (see introduction).

The copper-coated housings of the SDMs were replaced by carbon fiber composites to reduce ghosting artifacts resulting from eddy currents. They promise good shielding properties at high frequencies while having a reduced conductivity at the lower frequencies of the gradient switching [31]. The screens (details published in [32]) were formed as tubes and pushed over the modules. RF gaskets reduce the risk of leakage (Fig. 1, right).

\section{B. PET/RF Gantry and Infrastructure}

The preclinical PET gantry is composed of 10 SDMs (Fig. 2). It is built onto a trolley holding all system components that have to be brought into the MRI examination room.

1) Synchronization: The independent SDMs are synchronized with a 100-MHz reference clock (RefCLK), and a synchronization pulse (Sync) (used e.g., for a common counter reset and data acquisition start). Synchronization is performed as an optical system to improve MRI compatibility (see introduction). The combined RefCLK/Sync signal is generated by an FPGA

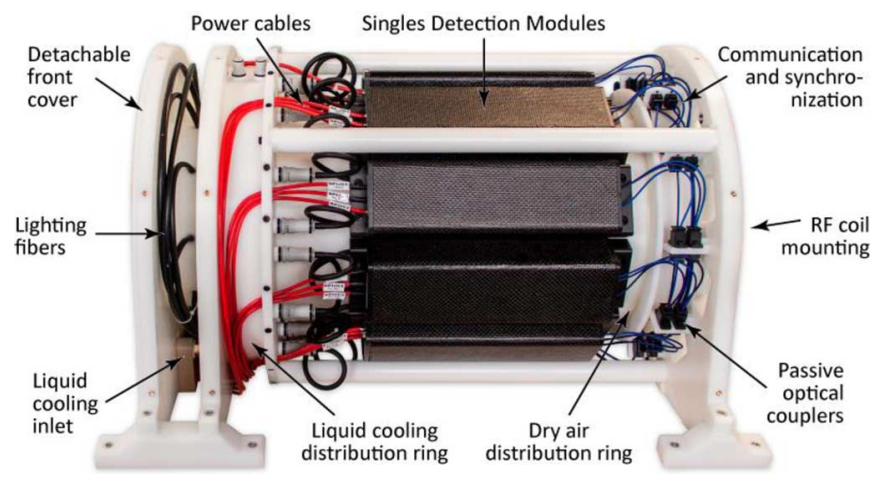

Fig. 2. Side view of the PET gantry. The SDMs are mounted on the cooling distribution rings. There is no further material between the SDMs and the RF coil to keep the attenuation for the gamma photons low. The infrastructure is connected to the SDMs at the gantry.

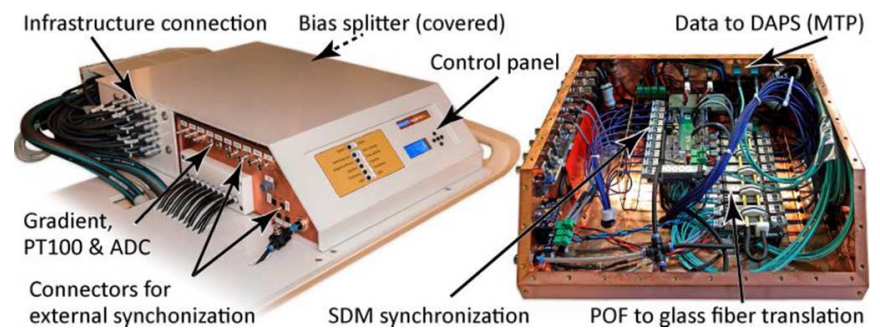

Fig. 3. The synchronization unit generates and distributes RefCLK/Sync signals for the SDMs. It furthermore provides synchronization to external devices and a user control interface. Communication to the SDMs is translated from POF to glass fiber.

in the synchronization unit (Fig. 3) and is distributed over POF transmitters to the SDMs.

The synchronization unit also offers multiple options for transferring trigger signals to and from other devices (galvanically and optically). Further available are: two Analog to Digital Converters (ADCs) (e.g., to sample respiratory information for PET motion correction [33]), three PT100 temperature sensor ports (e.g., to monitor animal heating devices), and an input for an MRI gradient switching detection coil (for temporal synchronization [34]). The housing of the synchronization unit also contains the conversion from POF to glass fiber communication, needed to connect to the standard optical gigabit Ethernet adaptors (Intel) in the DAPS. The glass fibers exit the examination room through a waveguide (see Suppl. fig. 3) in a single, non-conductive cable.

2) Power Supply: Power is brought to the SDMs with coaxial, partly semi-rigid, cables (Fig. 2), thanks to their favorable electromagnetic compatibility properties. To ensure that the current flowing to the SDMs in the cores of the cables is returned on the screens of the same cables, only one ground star point (the splitter for the bias voltage, see Fig. 4, right) is used for the complete system. A diagram of the power supply concept is displayed in Suppl. fig. 4. The power supply itself is placed on the insert trolley underneath the patient table. It is fully RF-screened to keep common-mode currents on the power cables low, as they can result in decreased SNR and spurious signals in the MR images [35]. All outputs of the power supply 


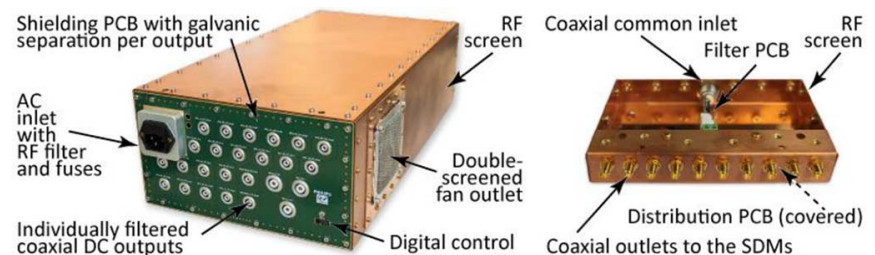

Fig. 4. Power supply: The power supply is placed in an RF screened housing (left).
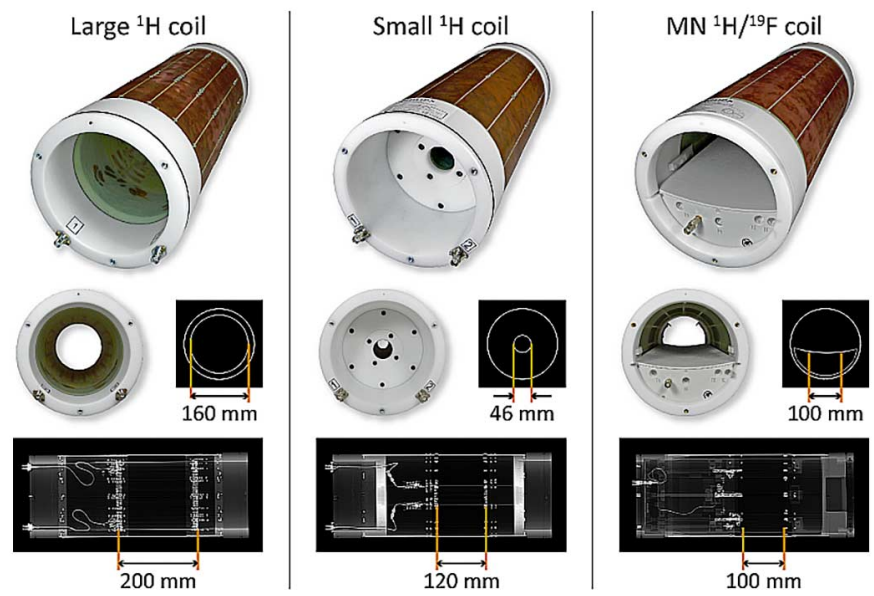

Fig. 5. Three gamma-transparent $\mathrm{Tx} / \mathrm{Rx} \mathrm{RF}$ coils, built for the insert: CT scans (transverse slice and coronal X-ray image) indicate the gamma transparency in the FOV.

are filtered inside the power supply housing (Fig. 4, left) with combined differential- and common-mode filters.

3) RF Coils: The MRI Transmit/Receive (Tx/Rx) RF coil is placed inside the PET gantry. As such, the coil is close to the subject to image (good for MRI SNR) and the cabling of the PET system is outside the exciting RF field (suppression of RF coupling between RF-Tx and PET supply cables). As the PET gamma photons have to travel through the coil, a gamma transparent design is required. Ceramic capacitors and thick wires were therefore removed from the FOV, and mechanical structures were thinned in the region of the PET FOV. The RF coil is exchangeable and three different coils have been built so far (Fig. 5). The large ${ }^{1} \mathrm{H}$ coil is a 16-rung high pass birdcage resonator with a diameter of $160 \mathrm{~mm}$ and a length of $200 \mathrm{~mm}$. It generates a maximum $\mathrm{B}_{1}$ field of $35 \mu \mathrm{T}$. The small ${ }^{1} \mathrm{H}$ coil, dedicated to mouse imaging, is made from a 12-rod-birdcage resonator with a diameter of $46 \mathrm{~mm}$ and a length of $120 \mathrm{~mm}$ (maximum $\mathrm{B}_{1}$ field is $30 \mu \mathrm{T}$ ). The third coil is a double-resonant Multi-Nuclei (MN) ${ }^{1} \mathrm{H} /{ }^{19} \mathrm{~F}$ coil (maximum $\mathrm{B}_{1}$ field of $20 \mu \mathrm{T}$ for both frequencies: $127.728 \mathrm{MHz}$ and $120.3 \mathrm{MHz}$ ). Unlike the other two coils, it is designed as an inductively coupled surface coil with a length and width of $100 \mathrm{~mm}$ [36]. All coils have an RF screen around the outer glass fiber composite cylinder. The screen is made from copper strips (18 $\mu \mathrm{m}$ thick) that are high-pass-coupled with capacitors to reduce eddy currents induced by the MRI gradients.

4) Installation: The PET/RF insert is mounted on an MR-compatible trolley, which docks to a patient table of a clinical Philips 3-T Achieva MRI system (Fig. 6). Power is taken

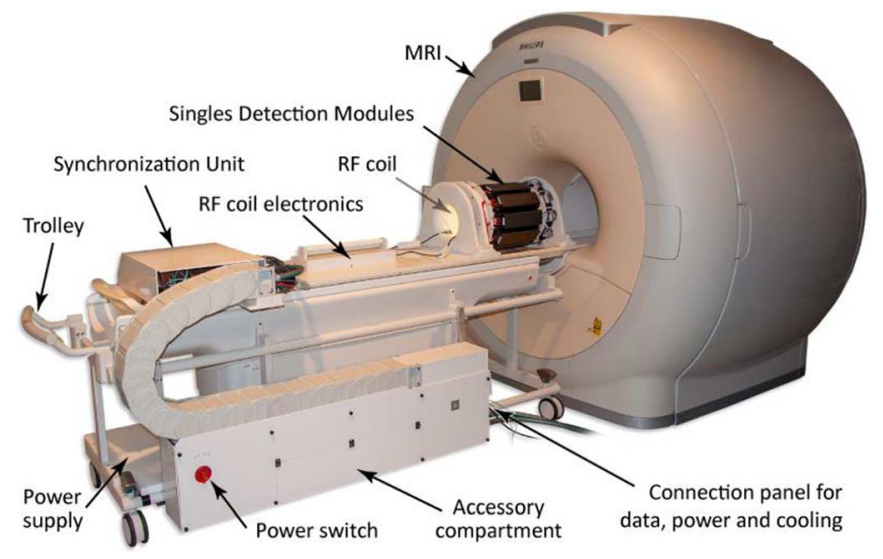

Fig. 6. The PET/RF insert "Hyperion $\mathrm{II}^{\mathrm{D}}$ " mounted on the patient table of a clinical $3 \mathrm{~T}$ MRI system (gantry cover removed).

TABLE II

SYSTEM DETAILS OF THE PET/MRI INSERT

\begin{tabular}{l|l}
\hline \hline Parameter & Value \\
\hline Total amount of detector stacks / crystals & $60 / 54000$ \\
Total amount of DPC dies / channels (pixel) & $960 / 3840$ \\
PET FOV $($ transaxial $\times$ axial) & $209.6 \mathrm{~mm} \times 96.6 \mathrm{~mm}$ \\
MRI FOV Large ${ }^{1} \mathrm{H}$ coil (transaxial $\times$ axial) & $160 \mathrm{~mm} \times 200 \mathrm{~mm}$ \\
MRI FOV Small ${ }^{1} \mathrm{H}$ coil (transaxial $\times$ axial) & $46 \mathrm{~mm} \times 120 \mathrm{~mm}$ \\
MRI FOV MN ${ }^{1} \mathrm{H} /{ }^{19} \mathrm{~F}$ coil (transaxial $\times$ axial) & $100 \mathrm{~mm} \times 100 \mathrm{~mm}$ \\
\hline \hline
\end{tabular}

a) crystal-to-crystal spacing

from an outlet in the MR examination room. The other three infrastructure cables $(2 \times$ cooling and data $)$ are not conductive and exit the shielded room through waveguides. Further details and dimensions are summarized in Table II and Suppl. fig. 5.

\section{PET System Back End}

1) Data Acquisition and Processing: The DAPS (Dell Poweredge R910) collects the data from the insert with six optical dual-port Ethernet adapters. Singles and coincidences are processed in real-time with software and list-mode data are saved [37]. Alternatively, the raw data are saved on the hard drives while the RAM is used as a buffer. Singles and coincidences processing uses sliding window techniques. The hits reported by the DPCs are first sorted and clustered at the detector stack level. Gamma crystal interaction position is then determined by a Center Of Gravity-Automatic Corner Extrapolation (COGACE) Anger algorithm [38]. Alternatively, a Maximum Likelihood (ML) method [39] with ML filtering (removing 30\% of the Lines Of Response (LOR) with the lowest likelihood values) is employed when stated. In this paper, a narrow energy window from $411 \mathrm{keV}$ to $561 \mathrm{keV}$ and a wide energy window from $250 \mathrm{keV}$ to $625 \mathrm{keV}$ (to include events that were Compton scattered in detector crystals and in large objects) are used. The coincidence window is set to $1 \mathrm{~ns}$.

2) Reconstruction: A list-mode image reconstruction algorithm is used with maximum likelihood expectation maximization including self-normalization and resolution modelling (OSEM-RM) [40]. The self-normalization can be performed directly on the measured data if the whole active area is included in the FOV (as is the case here), or otherwise an additional arbitrary scan satisfying aforementioned criteria can be used. 
Resolution modelling is achieved similar to [41]. Intra-crystal scattering has not been considered. In this paper, unless stated otherwise, the images are reconstructed in 32 iterations ( 16 for the in vivo images) with 8 subsets per iteration, and an isotropic voxel resolution of $0.25^{3} \mathrm{~mm}^{3}$. Although the reconstruction software is capable of attenuation- and scatter-correction, it was not used for the presented images (for accurate PET quantification, attenuation corrections should be employed [42]). Image fusion is achieved either manually or with AMIDE [43] and Imalytics [44].

3) Control and Calibration: The insert, as well as DAPS and the reconstruction server, is controlled on a workstation next to the MRI system console. Calibration of the system is performed at different times: for instance, a map showing the dark counts for all individual 12 million DPC cells was measured once after assembly. A calibration of time stamps (Time-to-Digital Converter of the DPCs) or crystal energy calibration factors are performed every time the system configuration was changed. The necessary calibration steps and chosen parameters depend on the optimization goal (e.g., highest sensitivity vs. highest possible activity). Throughout this paper, conservative settings were used: $20 \%$ of the cells were deactivated, $0^{\circ} \mathrm{C}$ or $5^{\circ} \mathrm{C}$ liquid cooling temperature, $2.5 \mathrm{~V} \mathrm{SiPM}$ cell overvoltage, trigger scheme 3 , validation network $0 \times 54$ or $0 \times 55$ [45].

\section{MeAsurement Methods}

\section{A. PET/MRI Interference Tests: Influence of PET on MRI}

1) $\mathrm{B}_{0}$-Distortion: $\mathrm{B}_{0}$-homogeneity was evaluated according to the "Magnetic Resonance Imaging Quality Control Manual" [46] $\mathrm{B}_{0}$ field maps were calculated from the phase differences of two scans with slightly different echo times. A 31 bottlephantom (mineral oil, a diameter of $140 \mathrm{~mm}$ ) was scanned with the large ${ }^{1} \mathrm{H}$ RF coil (isotropic voxel size of $2^{3} \mathrm{~mm}^{3}$ ) with and without automatic pencil beam volume shimming. The results from the operating PET/RF insert were compared to measurements made only with the RF coil and to the previous PET/RF insert.

2) Spurious Signals, SNR, and Image Uniformity: A built-in quality control batch scan from the MR system was used to detect unwanted spurious signals and noise in the receiving RF chain. It covers a total bandwidth of $730 \mathrm{kHz}$ with $180 \mathrm{~Hz} /$ pixel. The sequence was modified to suppress all RF transmission. The mean received signal over frequency was plotted by averaging the data in the phase encoding direction, which represent multiple measurements. The batch scans were executed with the two ${ }^{1} \mathrm{H}$ RF coils, while the PET system was in normal operation, measuring seven ${ }^{22} \mathrm{Na}$ point sources $(\sim 8.9 \mathrm{MBq}$ in total). References were acquired with the RF coils only.

Method 4 from the NEMA standards publication MS 1-2008 [47] is used to quantify the change in MRI image SNR. A 11 bottle-phantom (water, $\mathrm{CuSO}_{4}$, Arquad, $\mathrm{H}_{2} \mathrm{SO}_{4}$ ) with a diameter of $93 \mathrm{~mm}$ was imaged with a Spin Echo (SE) sequence $\left(\mathrm{TR} / \mathrm{TE}=612 \mathrm{~ms} / 20 \mathrm{~ms}\right.$, voxel size $=0.5^{2} \mathrm{~mm}^{2} \times 2 \mathrm{~mm}$, $320^{2}$ pixel, pixel bandwidth $=640 \mathrm{~Hz}, 3: 17$ min measurement time) while the PET insert measured the seven point sources placed around the bottle (PET processing with the wide energy window and reconstruction with isotropic voxel size of $0.5^{3} \mathrm{~mm}^{3}$ ). The SNR was calculated from one large signal region of interest (ROI) of the phantom and four ROIs in the corners, representing background noise (noise estimation via Rayleigh distribution fitting). The results were compared to an image of the same phantom arrangement, scanned by the large ${ }^{1} \mathrm{H}$ coil without the PET insert.

MRI image uniformity was accessed using the "ACR MR Accreditation Procedure", described in the NEMA standards publication MS 3-2008 [48]. The Percent Image Uniformity (PIU) was calculated from small averaged areas around the maximum and minimum intensity inside the phantom.

The experiments were repeated with a set of standard sequences: 2D Turbo Spin Echo (TSE) sequences, 3D Fast Field Echo (FFE) gradient echo sequences and multiple Echo Planar Imaging (EPI) sequences. Detailed information is displayed in Suppl. table I.

\section{B. PET/MRI Interference Tests: Influence of MRI on PET}

1) Sensitivity Degradation by the RF Coils: Gamma transparency of the RF coils was assessed by measuring a cylindrical ${ }^{22} \mathrm{Na}$ source (activity of $3.3 \mathrm{MBq}$, active volume of $4 \mathrm{~mm}$ diameter $\times 4 \mathrm{~mm}$ length, in a metal container with $6.3 \mathrm{~mm}$ diameter $\times 9 \mathrm{~mm}$ length). This was held in the center of the FOV by a 1-mm thick carbon fiber holder, attached to the outside of the insert. As such, the RF coils could be exchanged without moving the ${ }^{22} \mathrm{Na}$ source. Subsequent 3-minute-long PET measurements were made with and without all three RF coils. The closest distance of each detected LOR to the center of the source was determined, and the differences in count rates (introduced by the coils) were calculated as function of the distance. To estimate the loss in sensitivity caused by the coils, all LORs through a spherical volume-ROI around the source were counted. The selected ROI radius was $12 \mathrm{~mm}(10 \mathrm{~mm}$ as defined by NEMA NU 4 [49] for sensitivity calculations of point sources plus $2 \mathrm{~mm}$ accounting for the active source diameter).

2) PET Performance: To test the influence of the MRI scanner on the PET performance parameter, seven ${ }^{22} \mathrm{Na}$ point sources were placed in a transaxial, horizontal row and measured for 23 minutes. During that scan, three MRI sequences were executed: a $T_{1}$-weighted $\left(T_{1} w\right)$ TSE (using RF pulses for the echo trains), $\mathrm{T}_{1} \mathrm{w}$ 3D FFE (using gradients for the echoes) and an EPI sequence (using gradients with high slew rates (phase (X) and readout (Y): $203.8 \mathrm{mT} / \mathrm{m} / \mathrm{ms}$, slice selection (Z): $121.6 \mathrm{mT} / \mathrm{m} / \mathrm{ms}$ ) and switching duty cycles (X: $9.6 \%$, Y: $12 \%, \mathrm{Z}: 3.6 \%)$ ). Apart from small changes, such as the number of acquired slices to make the sequences about three minutes long, these did not differ from the sequences used in the SNR measurements. Details of the sequences are listed in Suppl. table II (measurements with altered sequences, not being able to produce images but having increased duty cycles to improve the statistical level, are presented in [26]). Temperatures on the SPUs and the temperatures of the DPCs were monitored with built-in digital sensors on the PCBs. Bias currents through the detector diodes were measured with ADCs on the interface boards. Singles and coincidences count rates were calculated in $0.1 \mathrm{~s}$ bins. Anger algorithm and the small energy window were used as they are potentially more sensitive to influences from the MRI. Liquid cooling temperature was set to $0^{\circ} \mathrm{C}$ 
for the same reason. PET photopeak position, energy, and time resolution were determined by fitting Gaussian functions in histograms over $5 \mathrm{~s}$ scan time (for fitting parameter see [38]). Spatial resolutions were measured as Full-Width at Half-Maximum (FWHM) by fitting a Gaussian function for each dimension over five high resolution sources with an active diameter of $0.25 \mathrm{~mm}$.

\section{PET/MR Imaging}

1) Hot-Rod Phantom: PET/MR images of a mouse-size hot-rod phantom were acquired using the small ${ }^{1} \mathrm{H}$ coil to visualize spatial resolutions and possible distortions. The hot-rod insert of the phantom measured $20 \mathrm{~mm}$ in height and $28 \mathrm{~mm}$ in diameter. It had six hot-rod arrays with rod diameters and gaps of $2,1.5,1.2,1.0,0.9$ and $0.8 \mathrm{~mm}$ (center-to-center spacing is two times the rod diameter). The phantom was filled with ${ }^{18} \mathrm{~F}$-Fluorodeoxyglucose $\left({ }^{18} \mathrm{~F}\right.$-FDG) having a total activity of $20.3 \mathrm{MBq}$ when a PET-only scan started (about $25 \%$ of the activity is in the rods). Directly after that reference scan, the PET insert was moved into the MRI and five subsequent PET/MRI scans were made. All PET measurements were stopped automatically, once 150 GByte of raw data were acquired (5:26 min for PET only and 8:05 min for the last scan with a start activity of $12.6 \mathrm{MBq}$ - details are listed in Suppl. table III). The narrow energy window and the ML algorithm were applied for singles processing. The MRI sequences were based on the sequences used in the SNR measurements. The acquisition pixel size was changed to $0.2^{2} \mathrm{~mm}^{2}$ for all imaging sequences (except for the $\mathrm{T}_{1} \mathrm{w} 3 \mathrm{D}-\mathrm{FFE}$ sequence that needs $0.3^{2} \mathrm{~mm}^{2}$ to keep the short echo time). Compared to the sequences used for the old insert, not only was the spatial resolution increased (acquisition pixel size for Hyperion I: $0.25^{2} \mathrm{~mm}^{2}$ and $0.3^{2}$ $\mathrm{mm}^{2}$ for $\mathrm{T}_{1} \mathrm{~W}$ 3D-FFE, $0.5^{2} \mathrm{~mm}^{2}$ for EPI), but the Number of Samples Averaged (NSA) was also halved to reduce the scan time. Details for all sequences are listed in Suppl. table IV. The MRI scans were repeated until the PET scan time was over. Due to the short scan time of the EPI sequences (from $3.3 \mathrm{~s}$ to $2 \mathrm{~s}$ ), these were repeated with different NSA values and echo train lengths.

2) $M N{ }^{1} H /{ }^{19} F$ Coil: To test the operation of the MN ${ }^{1} \mathrm{H} /{ }^{19} \mathrm{~F}$ coil, a phantom for coronal orientation $(82 \mathrm{~mm}$ outer diameter) was built. It was split into two compartments by a sagittal wall. One compartment and the outer ring $(3 \mathrm{~mm}$ thickness) were filled with water (approximately $20 \mathrm{~mm}$ high). Six vials with $1.8 \mathrm{ml}$ of different content were placed into both compartments, filled with isopropyl alcohol (IPA), standard phantom fluid $(\mathrm{PhF})$, air or water (depending on the compartment), ${ }^{18}$ F-FDG with an activity of approximately $5 \mathrm{MBq}$, perfluoro-15-crown-5-ether $\left(\mathrm{C}_{10} \mathrm{~F}_{20} \mathrm{O}_{5}\right)$ and olive oil. While PET data were acquired, multiple MR sequences (e.g., ${ }^{1} \mathrm{H}$ $\mathrm{T}_{1} \mathrm{w},{ }^{1} \mathrm{H} \mathrm{T}_{2} \mathrm{w}$, proton-density-weighted (PDw) TSE and ${ }^{19} \mathrm{~F}$ FFE) were executed. Fat suppression was used (Spectral Presaturation pulse with Inversion Recovery (SPIR) to generate a water-weighted image. From the available data, a fat-weighted image was calculated as the absolute difference from the PDw image. Details of the sequences are listed in Suppl. table V. The PET image was reconstructed from a five-minute-long data acquisition with MRI activity. PET and MR images had a reconstruction pixel size of $0.5^{2} \mathrm{~mm}^{2}$ and a slice thickness of $2 \mathrm{~mm}$.

3) In Vivo Measurements: A longitudinal study was performed to study the image quality and to prove the in vivo capabilities of the insert. All animal procedures were approved by the Maastricht University ethical review committee and were performed according to Dutch national law and the institutional animal care committee guidelines.

Human breast tumor cells (MDA-MB-231, $5 \times 10^{6}$ cells in $100 \mu 1$ PBS) were injected subcutaneously in the hind leg of an 8-week old female Balb/c mouse (Charles River, Burlington, MA USA) and the tumor size was monitored weekly with a caliper. The mouse was measured three times: 4, 16, and 37 days after the implantation. At each occasion it was anesthetized with isoflurane ( $3 \%$ induction, $1-2 \%$ maintenance) and placed in an MRI-compatible animal setup (Minerve, Esternay, France). The mouse was kept warm with hot air circulating inside the animal bed and its temperature was surveyed during the experiment with an optical rectal temperature sensor (Neoptix, Québec, Canada). Respiration was monitored with a pressure sensor (Rapid Biomedical, Rimpar, Germany). Three vials with small droplets of ${ }^{18} \mathrm{~F}-\mathrm{FDG}(\sim 500 \mathrm{kBq}$ in total) were placed next to the mouse as fiducial markers to facilitate and verify image fusion.

Before inserting the animal setup into the scanner (equipped with the small ${ }^{1} \mathrm{H}$ coil), approximately $13 \mathrm{MBq}$ of ${ }^{18} \mathrm{~F}$-FDG $(\sim 100 \mu l)$ was injected intravenously via the tail vein. The displayed PET images were reconstructed from PET data recorded after an uptake time of at least half an hour. Multiple MRI sequences with different contrasts were executed during the examinations, which took up to 190-minutes (for details see Suppl. table VI and Suppl. table VII).

In the last measurement, custom-made Electrocardiography (ECG) leads were connected to the front paws of the mouse with electroencephalography paste. ECG- and respiratory signals were analyzed with a preclinical trigger unit (Rapid Biomedical, Rimpar, Germany) and were optically transmitted to the MRI scanner and to the PET insert. As such, prospectively respiratory-gated and ECG-triggered MRI CINE images of the mouse heart were acquired ( $\sim 5$ min scan time). These were fused with images from PET data acquired at the same measurement, which was retrospectively gated with the respiratory and the ECG signal.

\section{RESUlts}

\section{A. Influence of PET on MRI}

1) $\mathrm{B}_{0}$-Distortion: With the $\mathrm{PET} / \mathrm{RF}$ insert the total $\mathrm{B}_{0}$ field is decreased by $5.7 \mathrm{ppm}$ (value calculated from $\mathrm{f}_{0}$ ) compared to the RF-coil-only case, which decreases the field by $0.2 \mathrm{ppm}$ compared to the integrated body coil (Hyperion I: $6.3 \mathrm{ppm})$. The coronal and transverse central slice of the $\mathrm{B}_{0}$-map (Fig. 7(a), and (b)) show that the homogeneity is mainly altered by a second-order distortion, which can be improved by the shim system of the MRI scanner (Fig. 7(c)). The plots in Fig. 7(d) show almost linear increases with the volume diameter until the spherical shapes of the ROIs are limited by the PET FOV. Without shimming, a maximum distortion 


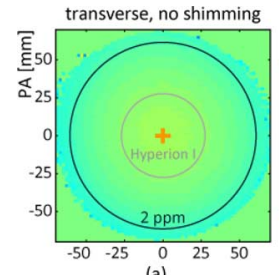

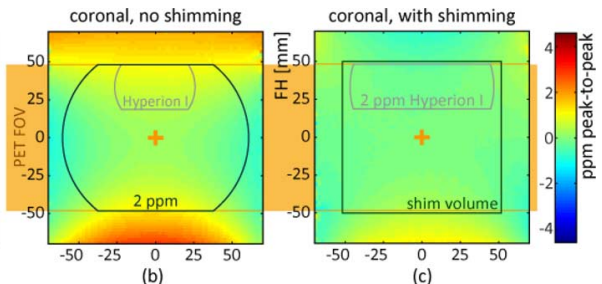

(b)

(c)

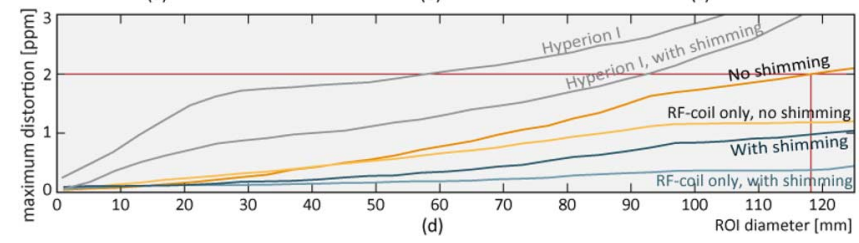

Fig. 7. $B_{0}$-maps: transverse center slice (a) and coronal center slice without (b) and with (c) shimming. The PET FOV is indicated in orange. The maximum peak-to-peak distortion for spherical ROIs, limited by the PET FOV, are plotted in (d) over the diameter. The smallest ROIs with a maximum distortion of $2 \mathrm{ppm}$ are indicated in all maps (in the shimming case, the whole map is below $2 \mathrm{ppm}$ ). For comparison, the results of the previous insert "Hyperion I" are overlaid in grey.

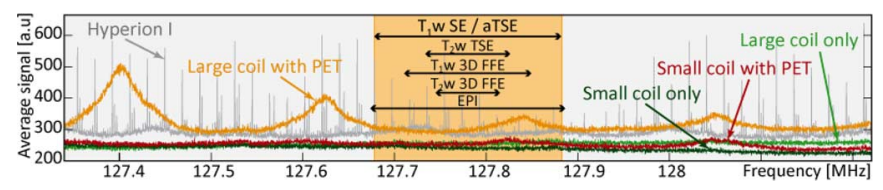

Fig. 8. Averaged MRI signal received by the ${ }^{1} \mathrm{H}$ RF coils over frequency. Bandwidths of the SNR sequences are indicated. The result of the old insert is overlaid in dark grey for comparison.

of $2 \mathrm{ppm}$ peak-to-peak is reached at a transaxial diameter of $118 \mathrm{~mm}$ (RF coil only: $132 \mathrm{~mm}$, Hyperion I: $56 \mathrm{~mm}$ ). With shimming, the distortion stays below $2 \mathrm{ppm}$ in the whole plotted map (Hyperion I: $90 \mathrm{~mm}$ ). At a diameter of $124 \mathrm{~mm}$, the maximum distortion is $1 \mathrm{ppm}$ peak-to-peak. Calculated as the VRMS value [50], the measured cylindrical volume in the hybrid FOV stays below $1.5 \mathrm{ppm}$ (without shimming). With shimming, the VRMS value stays below $0.1 \mathrm{ppm}$ in a spherical ROI with a diameter of $96 \mathrm{~mm}$ (Hyperion I: $8 \mathrm{~mm}$ ).

2) Spurious Signals, SNR, and Image Uniformity: The average MR signals over frequency, received by the two ${ }^{1} \mathrm{H}$ coils are shown in Fig. 8. The plot for the large coil shows distinct spurious signals at multiples of around $215 \mathrm{kHz}$, while the exact frequencies are not fixed, but shift with temperature and activity. Compared to RF-coil only, the mean received signal is increased by $28 \%$ from $253 \pm 7$ to $323 \pm 39$ (maximum value increased from 273 to 510 ). For the small coil the increase is $5 \%$ from $240 \pm 9$ to $253 \pm 9$ (maximum value from 264 to 278 ). Compared to the previous insert ( $292 \pm 26$ instead of $242 \pm 4$ for PET off) these values are slightly higher, but high amplitudes at localized frequencies (seen as spikes with a maximum value of 637 in Hyperion I plot) are not visible.

The increased noise floor results in a lower image SNR. Relative to MRI only, a decrease of $13 \%$ from 44.2 to 38 was calculated in the SE images presented in Fig. 9. That SNR loss is approximately the same factor as measured with the old insert (14\%). Nevertheless, the total SNR of 38 is increased by $80 \%$ compared to same measurement performed with Hyperion I (SNR of 21). The amplified backgrounds of the MR images are free from dotted stripes (zipper artifacts). The image uniformity changed $-1.9 \%$ from $89.8 \%$ (RF coil only) to $88.2 \%$ (PET/MRI).

The images produced by all sequences are presented in Suppl. fig. 6. A slight increase in background noise is visible in the PET/MR images, mainly in the EPI images (also shown in Fig. 9). The average SNR loss is $14 \%$ and the image uniformity is changed by $-1.7 \%$. Separate results for all sequences are displayed in Suppl. table VIII. Zipper artifacts are not noticeable in the images. EPI-typical artifacts are visible in particular with higher echo train length (see Fig. 9(e)). These are reduced compared to the old insert, whereas in Fig. 9(d), they were already clearly visible with EPI factors of eleven.

The point sources in the overlaid PET images of Fig. 9 are depicted slightly needle-shaped in a radial direction. The top point source (green rectangle in Fig. 9(b), shows zoomed region) is shown with a transaxial length of $1.9 \mathrm{~mm}$ (FWHM) and widths of $0.8 \mathrm{~mm} \times 0.9 \mathrm{~mm}$ (axial) (Hyperion I: $1.6 \mathrm{~mm} \times 1.8 \mathrm{~mm}$ $\times 1.3 \mathrm{~mm})$.

\section{B. Influence of MRI on PET}

1) Sensitivity Degradation by the RF Coils: The differences in detected count rates, compared to the coil-only case, are plotted in Fig. 10, right. They show that the count rates are reduced inside a radius of $4 \mathrm{~mm}$, whereas they are increased outside. Using the small energy window, the calculated sensitivity is reduced by a factor of $7.2 \%$ with the two ${ }^{1} \mathrm{H}$ coils and $8.4 \%$ with the $\mathrm{MN}$ coil. When the wide energy window is employed (Suppl. fig. 7), the sensitivity reduction factor is $5.6 \%$ for the large ${ }^{1} \mathrm{H}$ coil, $5.9 \%$ for the small ${ }^{1} \mathrm{H}$ coil, and $6.9 \%$ for the MN coil.

2) PET Performance: The results of the point source measurements during MRI sequences are displayed in Fig. 11. The averaged measured SPU temperature increases slightly during the gradient-intense EPI sequence. If there is a simultaneous increase in DPC sensor board temperature, it is lower than the normal fluctuations and total temperature drift during this long measurement. Nor are there any apparent changes during MRI activity to the bias currents, photopeak position, and count rates. The energy resolution is 12.6\% FWHM (Hyperion I: 29.7\%) and there are no indications that the applied MRI sequences led to degradations. Although the average time resolution of $565 \mathrm{ps}$ FWHM (Hyperion I: $2.5 \mathrm{~ns}$ ) only changes about 6 ps during the EPI sequence, it is more than 2.4 times the average standard deviation in the times without MRI activity and can thus be seen as an effect from the MRI on the PET system.

The spatial resolutions in X-, Y- (transaxial), and Z- (axial) directions are $0.9 \mathrm{~mm}$ in the center of the FOV. $40 \mathrm{~mm}$ in X-direction (half the PET FOV), the spatial resolutions were determined as $1.24 \mathrm{~mm}(\mathrm{X}), 0.8 \mathrm{~mm}(\mathrm{Y})$ and $0.97 \mathrm{~mm}(\mathrm{Z})$. The average measured volumetric spatial resolution in the center FOV is $0.73 \mathrm{~mm}^{3}$ (Hyperion I: $1.8 \mathrm{~mm}^{3}$ ). At higher radii, the volumetric spatial resolution decreases: at $40 \mathrm{~mm}$ it is $0.95 \mathrm{~mm}^{3}$ (Hyperion I: $2 \mathrm{~mm}^{3}$ )

\section{PET/MR Imaging}

1) Hot-Rod Phantom: Fig. 12 shows the images of the hot-rod phantom. The MR images are centered, but neither filtered nor resized (possible visual improvement of resolution 


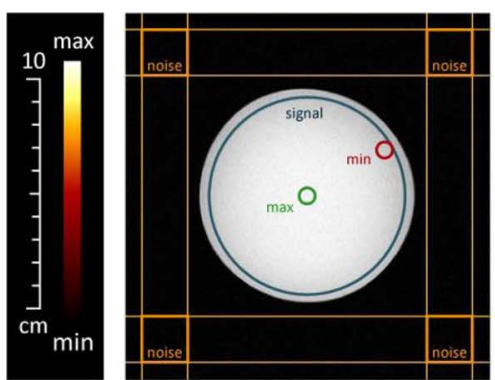

(a)

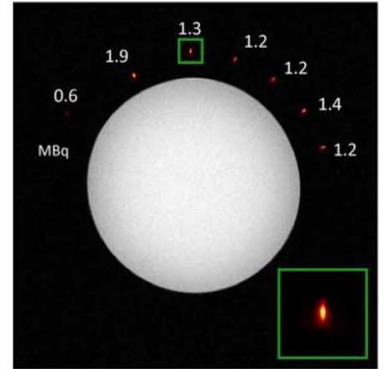

(b)

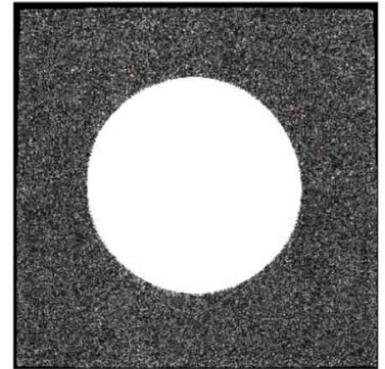

(c)

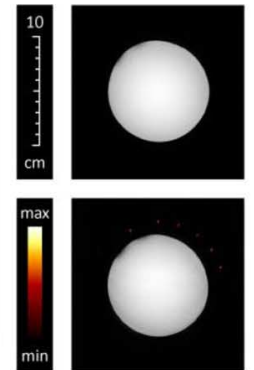

(d)

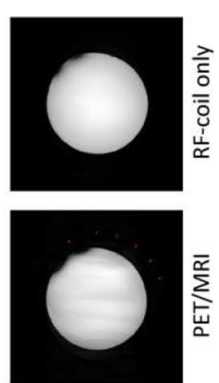

(e)

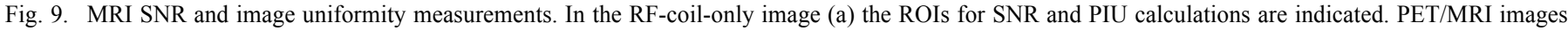

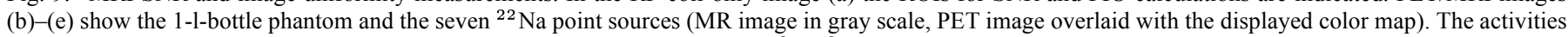

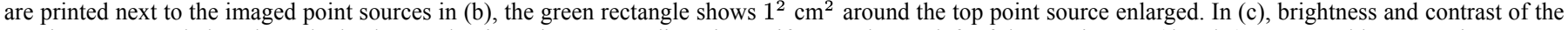

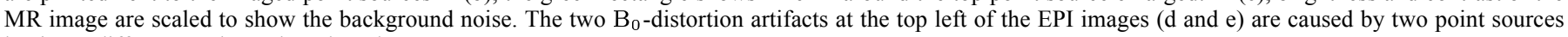
having a different package than the others.
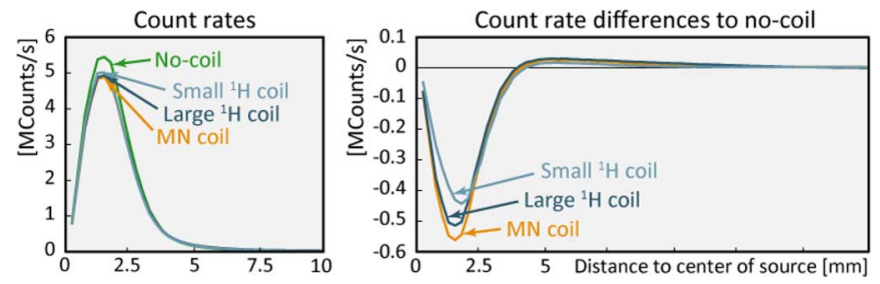

Fig. 10. Count rates and change in count rates (narrow energy window), caused by the three RF coils, over the distance to the center of the ${ }^{22} \mathrm{Na}$ source (wide energy window displayed in Suppl. Fig. 7).

by interpolation), nor are brightness and contrast adjusted manually. Visual differences between the MR images taken with and without PET cannot be ascertained. Nyquist ghosting artifacts, increasing with higher EPI factors, are visible in all EPI images, with and without the PET insert. Severe ghosting (where the ghosts have a similar intensity as the object) appeared in 15 of 266 images measured (Hyperion I: 14 of 184). This effect is independent of the presence of the PET insert (an example is shown in Suppl. fig. 8). Zipper artifacts are neither visible in the images nor in the backgrounds (the EPI 3 image (with PET) is shown again at the bottom right of Fig. 12, with size and brightness/contrast scaled to the background noise).

In the PET images, the polygonal shape of the detector geometry can be noticed in the shape of the outer ring of the phantom. Almost all rods (including the $0.8-\mathrm{mm}$ wide rods) are separable in all images (Hyperion I: 1-mm rods were blurred). Besides some statistical variations, there are neither differences between PET-only images and PET/MRI images, nor are artifacts visible. The line profiles through the $1.2-\mathrm{mm}$ and $0.9-\mathrm{mm}$ rods support this perception. The PET data from all scans were combined and reconstructed with a high-resolution voxel volume of $0.18^{3} \mathrm{~mm}^{3}$ (Fig. 13).

2) $M N{ }^{1} \mathrm{H} /{ }^{19} \mathrm{~F}$ Coil: Different coronal images of the phantom, sketched in Fig. 14(a), are shown in Fig. 14(b). The contrasts in the $\mathrm{T}_{1} \mathrm{w}{ }^{1} \mathrm{H}$ image (TR/TE: $600 \mathrm{~ms} / 31.5 \mathrm{~ms}$ ) and $\mathrm{T}_{2} \mathrm{~W}{ }^{1} \mathrm{H}$ (TR/TE: $2400 \mathrm{~ms} / 100 \mathrm{~ms}$ ) image are as expected: While the phantom fluid is enhanced in both images, fat (short $\mathrm{T}_{1}$ and a long $\mathrm{T}_{2}$ ) is bright in the $\mathrm{T}_{1} \mathrm{~W}$ image and water (long $\mathrm{T}_{1}$ and $\mathrm{T}_{2}$ ) and ${ }^{18} \mathrm{~F}$-FDG (solute in water) are bright in the
$\mathrm{T}_{2} \mathrm{w}$ scan. The water-fat-shift (one pixel, pixel bandwidth $445 \mathrm{~Hz}$ ) is visible for oil and isopropyl. The SPIR fat saturation works, the small susceptibility artifacts (in the water around the air vial and on the sides of the phantom) are most likely caused by the phantom itself. ${ }^{18} \mathrm{~F}-\mathrm{FDG}$ PET and ${ }^{19} \mathrm{~F}$ MR are free of visible artifacts.

For the combined image (Fig. 14(b)), PET, ${ }^{19} \mathrm{~F}$ and fat-weighted image were color mapped and overlaid (transparency according to brightness) over the water weighted image. The normalized profiles in Fig. 14(c) demonstrate that the four substances can be separated.

3) In Vivo Measurements: Fig. 15 shows images of the longitudinal study. Four days after cell injection the tumor had a volume of approximately $28 \mathrm{~mm}^{3}$. The tumor is visible in the $\mathrm{T}_{2} \mathrm{~W}$ MR image, but shows relatively low activity in the PET image. 12 days later the tumor measured $\sim 37 \mathrm{~mm}^{3}$, now clearly visible in the MR and in the PET image. Three weeks later the mouse was imaged for the last time after the tumor had grown within three days to approximately $42 \mathrm{~mm}^{3}$. Aside from the tumor, the PET image from day 16 shows high uptake in the kidneys, the brain, and Harderian glands behind the eyes. Although the brain is rather on the edge of the FOV and tracer injection was performed under anesthesia, some structure of the brain is already recognizable. Separated PET and MR images for all measurements are shown in Suppl. fig. 12.

The PET data from day 37 were gated with the information from the respiratory and ECG monitor. $47.6 \%$ of the 463.3 million coincidences were discarded due to respiratory and cardiac cycles reaching into the respiratory movements. The heart rate was relatively constant $( \pm 3.8 \%)$ at around 506 beats per minute. The data were binned once into four time bins and once into eight, all reconstructed independently. Fig. 16 shows the four time bins and small sections of the eight-time-bin version. The MR images are each combined from three (out of 15) time frames, representing the same time period as the four PET time bins (the full eight PET bins and single-MRI-time-frame images are shown in Suppl. fig. 13.) PET and MRI clearly show the contraction of the heart. Both ventricles are visible in the PET images and depicted without holes. An animation of the 8-time-bin version including all $15 \mathrm{MRI}$ time frames is presented in the supplementary material Suppl. fig. 14). 

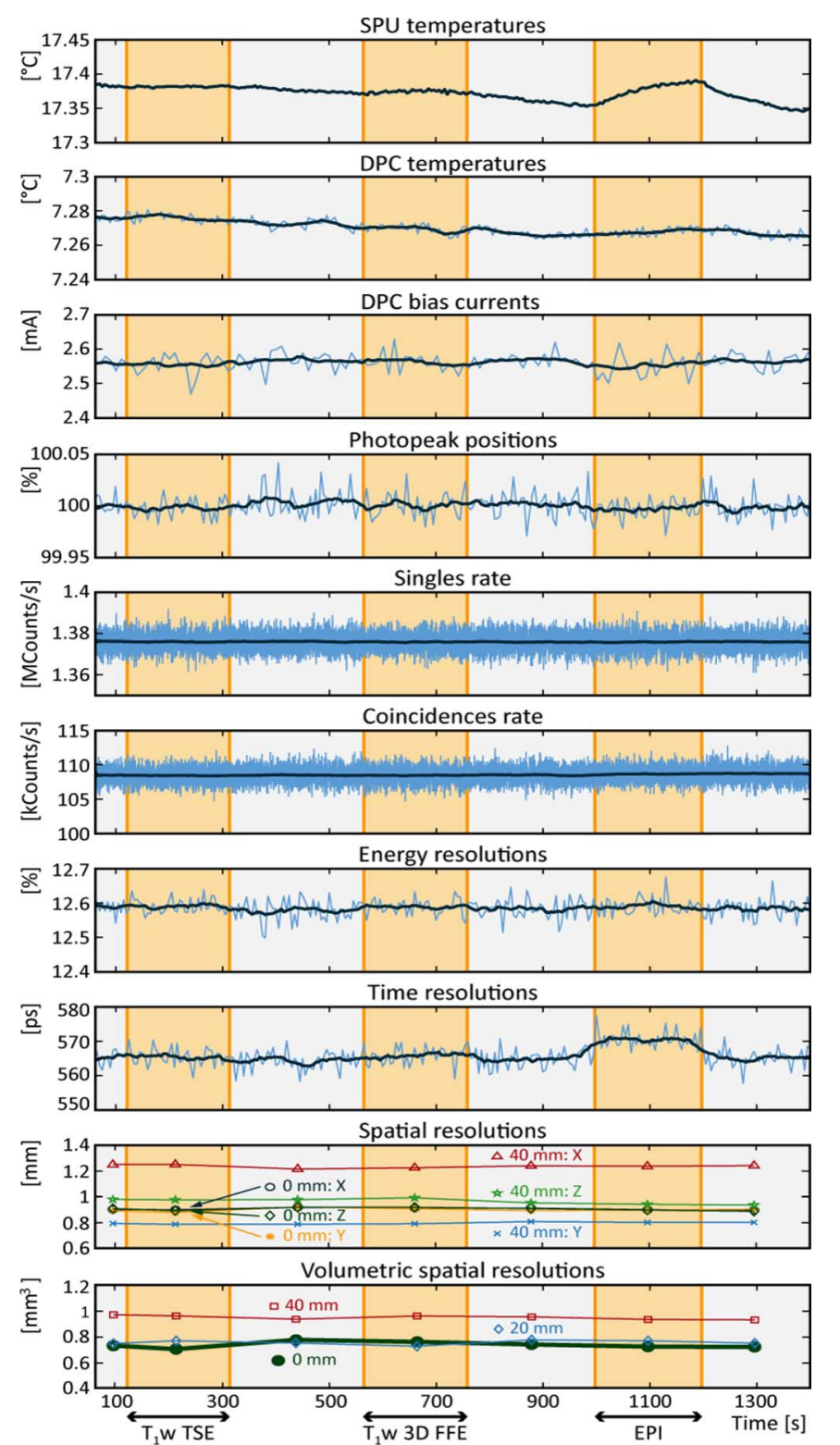

Fig. 11. Performance parameters of the PET insert during a 23-minute-long measurement of seven ${ }^{22} \mathrm{Na}$ point sources during which three different MR imaging sequences were executed (orange areas). The dark, thick curves are filtered with a 1-minute-moving-average filter to visualize tendencies. Spatial resolutions are plotted for a point source in the isocenter and at half the PET FOV (40 mm radius). Additionally, the volumetric spatial resolution is given for a slightly off-centered position (20 mm radius).

\section{Discussion}

\section{A. Influence of PET on MRI}

1) $\mathrm{B}_{0}$-Distortion: The overall change of $5.7 \mathrm{ppm}$ at the isocenter is of low relevance, since the resulting imaging frequency is calibrated in the preparation phase of each scan. The 2 ppm peak-to-peak distortion, described by [46] as a typical value over a 30 to $40 \mathrm{~cm}$ diameter sphere in clinical MRI scanner, is not exceeded in the hybrid ROI of $11 \mathrm{~mm}$ diameter. As the ratios of bore diameter to homogeneous ROI are similar, the $\mathrm{B}_{0}$-distortion is comparable to a clinical scanner without a PET insert. The full capacity of the shim system is still available to shim the field further disturbed by the objects to be scanned, since that value was measured without shimming. Whereas the $2 \mathrm{ppm}$ border can be seen as a good indication for imaging applications, MR Spectroscopy (MRS) has a much higher restriction to the homogeneity: a VRMS value of $0.1 \mathrm{ppm}$ should not be exceed in a single voxel (with a typical size of $20^{3} \mathrm{~mm}^{3}$ in humans) [46]. As $0.1 \mathrm{ppm}$ VRMS are kept within a ROI of $96 \mathrm{~mm}$ (with shimming) it allows single-voxel MRS even over complete organs of larger animals.

Although 60 detector stacks are installed compared to 20 for the old scanner, the diameter of the homogeneous ROI was enlarged from $56 \mathrm{~mm}$ to $118 \mathrm{~mm}$ (with shimming from $90 \mathrm{~mm}$ to the whole FOV). This demonstrates the positive impact of the measures described in Section II.A. Further improvements, if needed, can be achieved by applying e.g., active and passive shimming on PET detector level [51].

2) Spurious Signals, SNR, and Image Uniformity: The spurious signals, picked up by the large ${ }^{1} \mathrm{H}$ coil, show a pattern repeating with about $215 \mathrm{kHz}$ (Fig. 8). Its origin is the switchedmode power supply, which uses several switching frequencies in that range. The amplitude of the measured signal depends on the RF coil: compared to the old insert, the new large ${ }^{1} \mathrm{H}$ coil is more sensitive, resulting in a higher spurious signal pick-up (28\% increase instead of $21 \%$ increase), but also in higher overall SNR. The small ${ }^{1} \mathrm{H}$ coil is less sensitive to signals from the outside ( $5 \%$ increase compared to $28 \%$ increase) which is a consequence of its smaller dimensions and a lower RF-coupling between the screen and the birdcage resonator. Increased noise floors are frequently reported in PET/MRI research: [16] for instance, report an SNR loss between $53 \%$ and $80 \%$. The noise emitted by the PET detector electronics itself can be further reduced with techniques such as presented in [52]. Although the SNR reduction caused by the PET insert (13\%) is almost the same as for the previous insert (14\%), it can be seen as an improvement, because (due to the new RF coil) the total SNR measured in this experiment was increased by $80 \%$ compared to the old insert. Furthermore, the impact of $13 \%$ SNR reduction is limited, as it is in the same order of magnitude caused by intersubject variations [53]. Vertical stripes, visible in some images from the old insert (and often reported by other research groups [54], [55]), are neither noticeable in the images, nor in the backgrounds, nor in the spurious scans. Their disappearance shows that the new SDM shielding and the optical synchronization method are successful measures. The difference in integral image uniformity of $-1.9 \%$ between RF-coil only and PET/MRI is in the same order of magnitude as for the old insert $(-0.6 \%)$ and as results reported, for instance, in [56] (1.5\% to $6.5 \%)$ or $[53](0 \%$ to $-3.6 \%)$.

The Depth Of Interaction (DOI) effect, showing the point sources slightly needle-shaped at large radii (point sources in Fig. 9 and Suppl. fig. 6 are located at a radius of about $6 \mathrm{~cm}$ ), is intensified compared to the previous insert. This effect is mainly caused by the increased spatial resolution perpendicular to the radial direction, and is thus considered an improvement.

\section{B. Influence of MRI on PET}

1) Sensitivity Degradation by the RF Coils: The reduction of measured count rates inside a radius of $4 \mathrm{~mm}$, in combination 

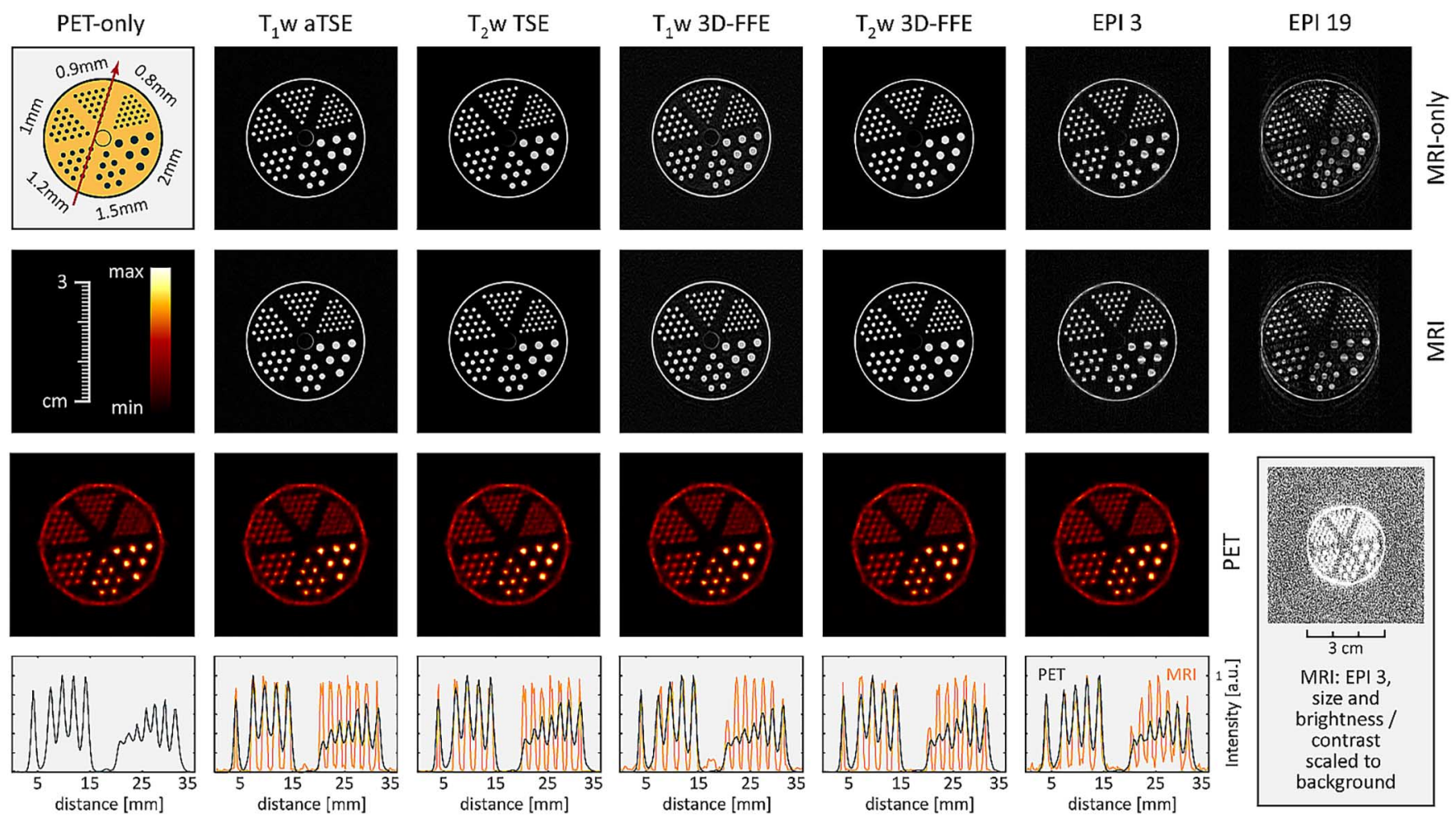

MRI: EPI 3,

size and

brightness /

contrast

scaled to

background

Fig. 12. PET only (left column), RF coil only (first row) and simultaneous PET/MRI measurements of the hot-rod. The orientation of the profiles (bottom row) through the simultaneously measured images (third row) is shown in the schematic of the phantom (top left). The image on the bottom right shows the MR image of the EPI 3 sequence (3.3 s scan time) with size and brightness/contrast scaled to show the background noise.
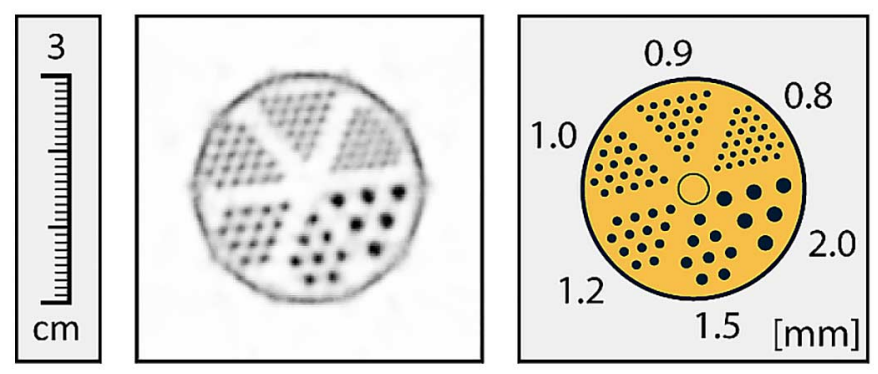

Fig. 13. High-resolution reconstruction $\left(0.18^{3} \mathrm{~mm}^{3}\right.$ voxel size $)$ of a combination of all PET datasets from Fig. 12, depicted in gray scale (profiles through all hot-rod areas are shown in Suppl. fig. 9.)

with the increased count rates close to that, is a clear indication of scatter introduced by the coils. More of these scattered events are accepted by the wider energy window, as Suppl. fig. 7 , right shows increased count rates at higher radii compared to the narrow window. Although these additional LORs decrease the loss in sensitivity (from $7.2 \%$ to $5.6 \%$ for the large ${ }^{1} \mathrm{H}$ coil), they also cause blurring of the PET image. A sensitivity reduction factor of $7.2 \%(8.4 \%$ for the $\mathrm{MN}$ coil) is significant, but, besides slightly lower precision due to higher noise, quantification of PET images is not affected, as attenuation maps of the coils can be used (the coils are always placed in the exact same position, which is ensured by rubber gaskets and a key-and-slot system with a mechanical tolerance of $-100 \mu \mathrm{m}$ ).

2) PET Performance: The heating of the SPU during the long gradient intense EPI sequence (Fig. 11) was also observed with the previous insert. Compared to that, mainly due to the improved cooling system, heating is about $50 \%$ lower, while cooling after the sequence is twelve times faster. Furthermore, the temperature of the sensors was stabilized and changes in bias currents, photopeak positions and count rates were no longer visible. Therefore, sensitivity can be considered stable during MRI activity, which is important for quantitative and dynamic studies.

The loss of time resolution (here $6 \mathrm{ps}$ on average) has been reported before [26] and is caused by induced voltages on the power supply network. After further investigation, the problem can be solved with updated hardware. Nevertheless, the measured effect is only $1 \%$. Besides allowing small coincidence time windows, and thus reducing the random rate, the TOF information can reduce the statistical noise in PET images [57]. With a time resolution of 565 ps (trigger scheme 3 ) the position error is $85 \mathrm{~mm}$ and TOF benefit is thus restricted to rabbit-sized subjects. With optimized settings for timing (trigger scheme 1 and $-5^{\circ} \mathrm{C}$ cooling temperature, see Suppl. fig. 10) time resolutions of $260 \mathrm{ps}$ could be achieved ( $240 \mathrm{ps}$ were shown with two modules, equipped with $4 \mathrm{~mm} \times 4 \mathrm{~mm} \times 10 \mathrm{~mm}$ crystals [58]). All these results demonstrate that the improved PET system with dSiPMs and optical synchronization method is suitable for TOF-PET under MRI conditions.

The spatial resolution of $0.9 \mathrm{~mm}\left(0.73 \mathrm{~mm}^{3}\right.$ volumetric spatial resolution) in the center of the FOV is not changed during MRI activity and outperforms the predecessor Hyperion I (transaxial: $1.2 \mathrm{~mm} \times 1.3 \mathrm{~mm}$, axial: $1.15 \mathrm{~mm}$, volumetric: $\left.1.8 \mathrm{~mm}^{3}\right)$.

A performance comparison between several preclinical PET and PET/MR prototypes can be found in [4]. First experiments 


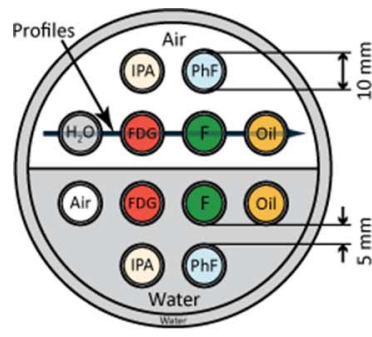

(a)

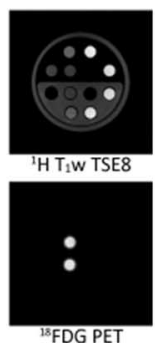

${ }^{3}$ FDG PET

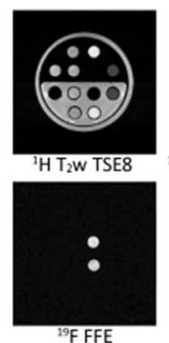

(b)

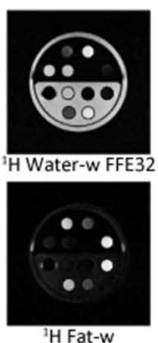

H Fat-w

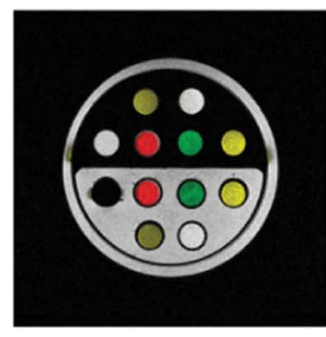

(c)

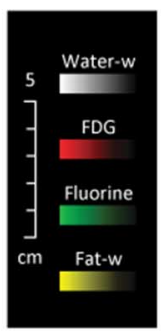

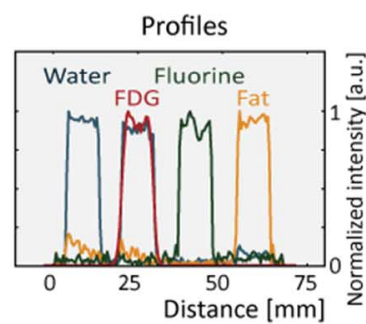

(d)

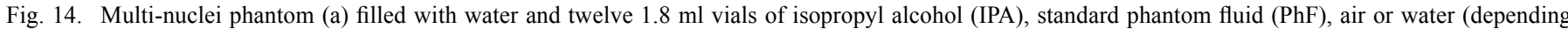

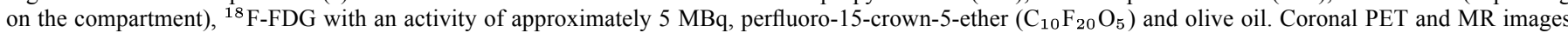

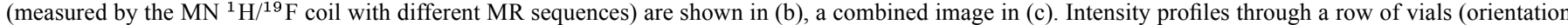
indicated in (a)) are plotted in (c).

indicate that peak sensitivity of the presented system is around $2.6 \%$, which is in the same range as the best PET/MR systems shown in this comparison, while the volumetric spatial resolution exceeds all analyzed PET/MR systems and PET standalone systems.

Since solid state detectors are known to work at higher magnetic field strengths than $3 \mathrm{~T}$ [11], the demonstrated technology should also work in combination with a 7-T (or higher) MRI scanner.

\section{PET/MR Imaging}

1) Hot-Rod Phantom: The phantom studies demonstrate that the MR images do not change their appearance with the PET gantry being present and acquiring data. As a result of the exchangeable RF coil and by using the small ${ }^{1} \mathrm{H}$ coil, the SNR was largely improved compared to the previous insert. PET/MR imaging examples with the large ${ }^{1} \mathrm{H}$ coil are shown in Suppl. fig. 11. Despite the high spatial resolution of $0.195 \mathrm{~mm}$, the EPI 19 sequence has a scan time of only $2 \mathrm{~s}$ (the EPI 3 needs $3.3 \mathrm{~s}$ ), which shows that high speed imaging (as needed e.g., for fMRI applications) is possible. Resolving the $0.8-\mathrm{mm}$ hot rods in the PET images is a large improvement over the previous insert, where even $1 \mathrm{~mm}$ rods were blurred. The ability to separate 0.8 $\mathrm{mm}$ hot rods with PET was shown before with a preclinical sequential 1-T MRI and PET system [9] using position-sensitive photomultiplier tubes, which cannot be used in a simultaneous configuration.

2) $M N{ }^{1} H /{ }^{19} F$ Coil: The images presented in Fig. 14 demonstrate, that the combination of the PET insert with the $\mathrm{MN}{ }^{1} \mathrm{H} /{ }^{19} \mathrm{~F}$ coil works as designed. Neither PETnor MR images show visible artifacts. This indicates that the shielding of all components is not limited to the MR frequency of ${ }^{1} \mathrm{H}$ at $3 \mathrm{~T}$, but also sufficient at the MR frequency of ${ }^{19} \mathrm{~F}$. The profiles shown in Fig. 14(c) demonstrate the differentiability between the scanned fluids and thus the adequate SNR of the methods applied.

3) In Vivo Measurements: The presented simultaneous measured PET/MR images in Fig. 15 show a high level of detail over the complete body of the mouse: from the tumor in the leg to structures in the kidneys and the brain. For quantification of the PET images, a calibration with a decaying source has to be performed (the stability of the system with respect to quantification will be the subject of future investigations). Here,

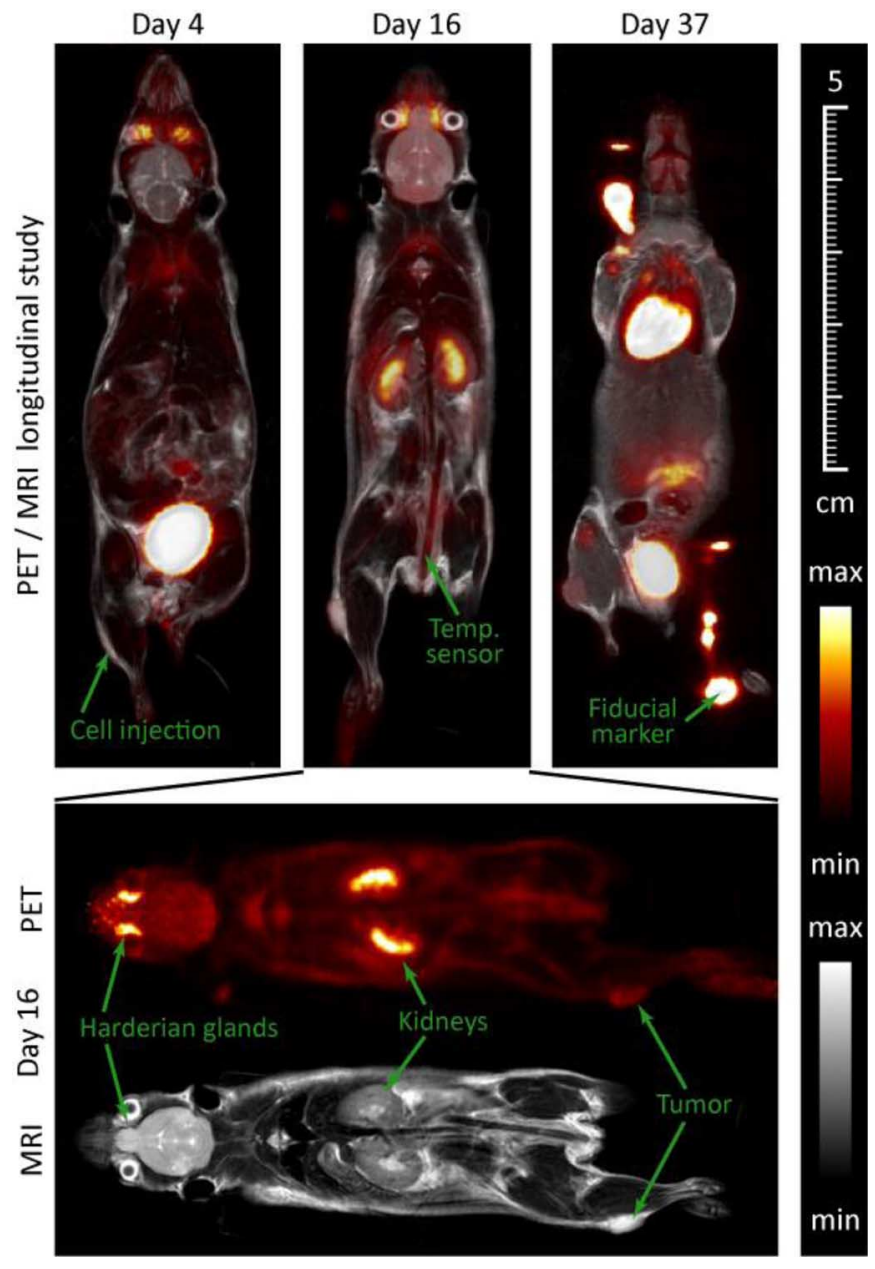

Fig. 15. Longitudinal study of a human breast cancer tumor growing subcutaneously on the left hind leg of a mouse. Top row shows simultaneously measured whole-body PET/MR images at 4 days after subcutaneous cell injection ( $1 \mathrm{~mm}$ slice $\left(0.195^{2} \mathrm{~mm}^{2}\right.$ pixel size $)$ of 24 multi-slice $\mathrm{T}_{2} \mathrm{~W}$ TSE16 sequences $(\mathrm{TR} / \mathrm{TE}=2.4 \mathrm{~s} / 0.1 \mathrm{~s})$, NSA2, 10:12 min scan time); after 16 days $\left(2 \mathrm{~mm}\right.$ single slice $\left(0.195^{2} \mathrm{~mm}^{2}\right.$ pixel size) $\mathrm{T}_{2} \mathrm{~W}$ TSE16 sequence $(\mathrm{TR} / \mathrm{TE}=2.4 \mathrm{~s} / 0.1 \mathrm{~s})$, NSA4, 5:03 min scan time); and after 37 days ( $1 \mathrm{~mm}$ slice $\left(0.195^{2} \mathrm{~mm}^{2}\right.$ pixel size) of 24 multi-slice $\mathrm{T}_{1} \mathrm{~W}$ TSE9 sequences $(\mathrm{TR} / \mathrm{TE}=612 \mathrm{~ms} / 20 \mathrm{~ms}), \mathrm{NSA} 4$, 5:03 min scan time). Below that, PET and MR images are shown separately for day 16 (day 4 and 37 are also displayed in Suppl. fig. 12).

quantification can be realized by normalizing the image on the total injected activity, as the whole mouse and fiducial marker are inside the FOV. The same animal was measured three times, 


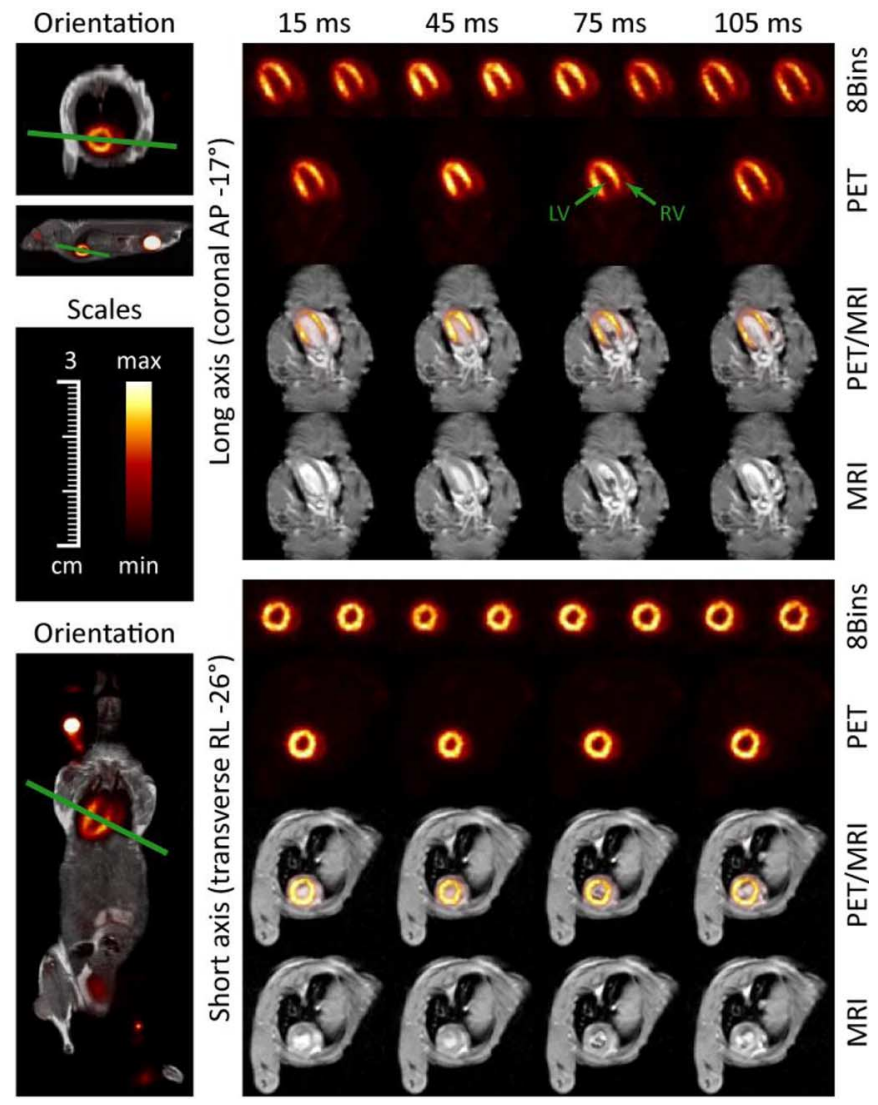

Fig. 16. Dual-gated (respiratory and ECG) simultaneous PET/MR measurement of a mouse heart. Four time bins are shown as PET, as MRI and as combined PET/MR images. Additionally, smaller sections of the PET images from 8 time bins are shown. The slices are orientated along the long (top) and short axis (bottom) of the heart. MRI gradient echo CINE sequence with $0.3^{2} \mathrm{~mm}^{2}$ pixel size and $1 \mathrm{~mm}$ slice thickness, $\mathrm{TR} / \mathrm{TE}=7.46 \mathrm{~ms} / 2.754 \mathrm{~ms}, \mathrm{FA}=10^{\circ}$ Both, left ventricle (LV) and right ventricle (RV) are visible in the PET images.

which demonstrates the in vivo capabilities of the insert as well as its usability for longitudinal studies.

The simultaneously measured, dual-gated PET/MRI CINE images of the mouse heart shows that the insert is suitable for more advanced preclinical research applications, requiring external equipment to be connected. PET and MRI images are well aligned, both temporally and spatially, which demonstrates the advantage of simultaneous data acquisition. The cardiac PET images show a good uniformity in the myocardial wall, and also the right ventricle of the mouse heart is visible. The presented measurements are directly required in preclinical cardiac applications, as described in [4], [59], and [60].

\section{CONCLUSION}

The preclinical PET/RF insert "Hyperion $\mathrm{II}^{\mathrm{D}}$ " is the world's first PET/MRI system using digital silicon photomultiplier technology. A range of improvements have been made over its predecessor with analog SiPMs and a digitization in the detector module: The hybrid FOV has been enlarged to $160 \mathrm{~mm} \times 96.6$ $\mathrm{mm}$ (transaxial $\times$ axial). The diameter of a spherical ROI within that FOV with a $\mathrm{B}_{0}$ disturbance lower than $2 \mathrm{ppm}$ peak-to-peak without shimming has been increased to $118 \mathrm{~mm}$. Within a diameter of $96 \mathrm{~mm}, 0.1 \mathrm{ppm}$ VRMS $\mathrm{B}_{0}$-homogeneity is kept employing automatic volume shimming. MRI Tx/Rx RF coils are now changeable and three different dedicated coils are already available. Using the large ${ }^{1} \mathrm{H}$ coil, the total MRI SNR has been improved by $80 \%$ compared to the previous insert. With the small ${ }^{1} \mathrm{H}$ coil, dedicated to mouse imaging, the PET system increases the MRI noise floor only by 5\%. Dotted lines (zipper artifacts) are neither visible in the MRI images nor in their amplified backgrounds. Except for a decrement in time resolution of 6 ps during an EPI sequence, multiple tested MRI sequences did not influence the measured PET performance parameter. With trigger scheme three, the time resolution is 565 ps (FWHM), the energy resolution is $12.6 \%$ (FWHM), and the spatial resolution is $0.9 \mathrm{~mm}$ in the isocenter (FWHM, $0.73^{3} \mathrm{~mm}^{3}$ volumetric). Using timing-optimized settings (trigger scheme one), the time resolution improves to $260 \mathrm{ps}$ (FWHM). PET images of the mouse-sized hot-rod phantom depict the 0.8 -mm hot-rods distinguishable, with and without MRI operation. Due to the high SNR of the small ${ }^{1} \mathrm{H}$ coil, EPI images of that phantom with a spatial resolution of $0.195^{2} \mathrm{~mm}^{2}$ are taken in $2 \mathrm{~s}$ and show no differences to images measured without the PET insert. First ${ }^{1} \mathrm{H} /{ }^{19} \mathrm{~F} /{ }^{18} \mathrm{~F}$ images, taken with the $\mathrm{MN}{ }^{1} \mathrm{H} /{ }^{19} \mathrm{~F}$ coil, demonstrate the versatility of the PET/RF insert, and that the RF tightness is not limited to the ${ }^{1} \mathrm{H}$ frequency at $3 \mathrm{~T}$. Repeated simultaneous whole-body mouse PET/MRI measurements show the operability and stability of the insert and demonstrate the usability in longitudinal in vivo studies. The dual-gated CINE heart images show that the insert is eligible for more advanced research applications and underline the advantages of simultaneous data acquisition.

The presented PET/MRI insert demonstrates that dSiPMs can be used to build high-resolution preclinical PET systems, and with an elaborated system design can also unfold their potential during simultaneous PET and MRI acquisition.

\section{ACKNOWLEDGMENT}

The authors thank Wolfgang Renz and Katharina Schumacher (formerly Irmato Industrial Solutions Aachen) for the insert mechanics and Daniel Wirtz (Philips Research Hamburg) for the small and large RF coils. The ${ }^{1} \mathrm{H} /{ }^{19} \mathrm{~F}$ coil was built by Christian Findeklee (Philips Research Hamburg) with the funding of InnoMeT (grant number z0909im008a). The authors gratefully thank Suzanne Kivits (formerly Philips Research Eindhoven) for ${ }^{18} \mathrm{~F}-\mathrm{FDG}$ phantom filling and Caren van Kammen (formerly Maastricht University) for animal handling. Furthermore, the authors thank Andreas Goedicke (Philips Research Eindhoven) for making CT scans of the RF coils possible.

\section{REFERENCES}

[1] D. W. Townsend, "Multimodality imaging of structure and function," Phys. Med. Biol., vol. 53, no. 4, pp. R1-R39, Feb. 2008.

[2] T. Beyer et al., "A combined PET/CT scanner for clinicaloncology," $J$. Nucl. Med., vol. 41, no. 8, pp. 1369-1379, Aug. 2000.

[3] H. F. Wehrl et al., "Simultaneous PET-MRI reveals brain function in activated and resting state on metabolic, hemodynamic and multiple temporal scales," Nat. Med., vol. 19, no. 9, pp. 1184-1189, 2013.

[4] M. S. Judenhofer and S. R. Cherry, "Applications for preclinical PET/ MRI," Semin. Nucl. Med., vol. 43, no. 1, pp. 19-29, Jan. 2013.

[5] D. A. Torigian et al., "PET/MR imaging: Technical aspects and potential clinical applications," Radiology, vol. 267, no. 1, pp. 26-44, 2013.

[6] H. F. Wehrl, A. W. Sauter, M. R. Divine, and B. J. Pichler, "Combined PET/MR: A technology becomes mature," J. Nucl. Med., vol. 56, no. 2, pp. 165-168, 2015.

[7] S. Vandenberghe and P. K. Marsden, "PET-MRI: A review of challenges and solutions in the development of integrated multimodality imaging," Phys. Med. Biol., vol. 60, pp. R115-R154, 2015. 
[8] P. Maniawski, "PET/MR: The molecular imaging dream team," Nucl. Med. Rev. Cent. East. Eur., vol. 14, no. 1, pp. 47-50, Jan. 2011.

[9] K. Nagy et al., "Performance evaluation of the small-animal nanoScan PET/MRI system," J. Nucl. Med., vol. 54, pp. 1825-1832, 2013.

[10] Y. Shao et al., "Simultaneous PET and MR imaging," Phys. Med. Biol., vol. 42, no. 10, pp. 1965-1970, Oct. 1997.

[11] B. J. Pichler et al., "Performance test of an LSO-APD detector in a 7-T MRI scanner for simultaneous PET/MRI," J. Nucl. Med., vol. 47, no. 4, pp. 639-647, Apr. 2006.

[12] M. S. Judenhofer et al., "Simultaneous PET-MRI: A new approach for functional and morphological imaging," Nat. Med., vol. 14, no. 4, pp. 459-465, Apr. 2008.

[13] G. Delso et al., "Performance measurements of the Siemens mMR integrated whole-body PET/MR scanner," J. Nucl. Med., vol. 52, no. 12, pp. 1914-1922, Dec. 2011.

[14] E. Roncali and S. R. Cherry, "Application of silicon photomultipliers to positron emission tomography," Ann. Biomed. Eng., vol. 39, no. 4, pp. 1358-1377, Apr. 2011

[15] V. Schulz et al., "A preclinical PET/MR insert for a human scanner," in IEEE Nucl. Sci. Symp. Med. Imag. Conf. Rec., 2009, pp. $2577-2579$.

[16] K. J. Hong et al., "A prototype MR insertable brain PET using tileable GAPD arrays," Med. Phys., vol. 40, no. 4, p. 042503, Apr. 2013.

[17] W. E. Timms, "Interference problems and screening in NMR and MRI," J. Med. Eng. Technol., vol. 16, no. 2, pp. 69-78, 1992.

[18] C. S. Levin et al., "Performance of a high sensitivity time-of-flight PET ring operating simultaneously within a 3T MR system," EJNMMI Phys., vol. 1, no. July, p. 7364, 2014.

[19] J. Schenck, "The role of magnetic susceptibility in magnetic resonance imaging: MRI magnetic compatibility of the first and second kinds," Med. Phys., vol. 23, no. 6, pp. 815-850, 1996.

[20] D. Le Bihan, C. Poupon, A. Amadon, and F. Lethimonnier, "Artifacts and pitfalls in diffusion MRI," J. Magn. Reson. Imag., vol. 24, no. 3, pp. 478-488, Sept. 2006.

[21] J. P. Hornak, The basics of MRI 2011 [Online]. Available: http://www. cis.rit.edu/htbooks/mri

[22] B. Weissler et al., "MR compatibility aspects of a silicon photomultiplier-based PET/RF insert with integrated digitisation," Phys. Med. Biol., vol. 59, no. 17, pp. 5119-5139, 2014.

[23] C. Degenhardt et al., "The digital silicon photomultiplier-A novel sensor for the detection of scintillation light," in IEEE Nucl. Sci. Symp. Med. Imag. Conf. Rec., 2009, pp. 2383-2386.

[24] Y. Haemisch, T. Frach, C. Degenhardt, and A. Thon, "Fully digital arrays of silicon photomultipliers ( $\mathrm{dSiPM})$ - A scalable alternative to vacuum photomultiplier tubes (PMT)," Phys. Procedia, vol. 37, pp. 1546-1560, 2012.

[25] B. Weissler et al., "Design concept of world' s first preclinical PET/MR insert with fully digital silicon photomultiplier technology," in IEEE Nucl. Sci. Symp. Med. Imag. Conf. Rec., 2012, pp. 2113-2116.

[26] J. Wehner et al., "MR-compatibility assessment of the first preclinical PET-MRI insert equipped with digital silicon photomultipliers," Phys. Med. Biol., vol. 60, pp. 2231-2255, 2015.

[27] J. E. Mackewn et al., "PET performance evaluation of a pre-clinical SiPM based MR-compatible PET scanner," IEEE Trans. Nucl. Sci., vol. 62, no. 3, pp. 784-790, Jun. 2015.

[28] P. M. Dueppenbecker et al., "Development of an MRI compatible digital SiPM based PET detector stack for simultaneous preclinical PET/ MRI," in IEEE Nucl. Sci. Symp. Med. Imag. Conf. Rec., 2012, pp. 3481-3483.

[29] B. Weissler, P. Gebhardt, M. Zinke, F. Kiessling, and V. Schulz, “An MR-compatible singles detection and processing unit for simultaneous preclinical PET/MR," in IEEE Nucl. Sci. Symp. Med. Imag. Conf. Rec., 2012, pp. 2759-2761.

[30] P. Gebhardt et al., "FPGA-based singles and coincidences processing pipeline for integrated digital PET/MR detectors," in IEEE Nucl. Sci. Symp. Med. Imag. Conf. Rec., 2012, pp. 2479-2482.

[31] D. D. L. Chung, "Electromagnetic interference shielding effectiveness of carbon materials," Carbon N. Y., vol. 39, no. 2, pp. 279-285, Feb. 2001.

[32] P. M. Dueppenbecker et al., "Gradient transparent RF housing for simultaneous PET/MRI using carbon fiber composites," in IEEE Nucl. Sci. Symp. Med. Imag. Conf. Rec., 2012, pp. 3478-3480.

[33] C. Tsoumpas et al., "Simultaneous PET-MR acquisition and MR-derived motion fields for correction of non-rigid motion in PET," Ann. Nucl. Med., vol. 24, pp. 745-750, 2010.

[34] B. Weissler et al., "PET/MR synchronization by detection of switching gradients," IEEE Trans. Nucl. Sci., vol. 62, no. 3, pp. 650-657, Jun. 2015 .
[35] J. Wehner et al., "PET/MRI insert using digital SiPMs: Investigation of MR-compatibility," Nucl. Instrum. Methods Phys. Res. Sect. A Accel. Spectrometers, Detect. Assoc. Equip., vol. 734, pp. 116-121, Jan. 2014

[36] C. Findeklee, C. Leussler, D. Wirtz, and J. Keupp, "Highly efficient inductively coupled double resonant surface coil for simultaneous 1H/19F PET-MRI," in Proc. ISMRM, 2013, vol. 30, no. 1, p. 0828.

[37] B. Goldschmidt et al., "Towards software-based real-time singles and coincidence processing of digital PET detector raw data," IEEE Trans. Nucl. Sci., vol. 60, no. 3, pp. 1550-1559, Jun. 2013.

[38] D. Schug et al., "Data processing for a high resolution preclinical PET detector based on Philips DPC digital SiPMs," IEEE Trans. Nucl. Sci., vol. 62, no. 3, pp. 669-678, 2015.

[39] C. Lerche, S. Lodomez, J. Wehner, B. Goldschmidt, and V. Schulz, "Likelihood based inter-crystal Compton scatter and pulse pile-up rejection for MR compatible preclinical PET insert," in World Mol. Imag. Soc., 2013, p. 607.

[40] A. Salomon, B. Goldschmidt, R. Botnar, F. Kiessling, and V. Schulz, "A self-normalization reconstruction technique for PET scans using the positron emission data," IEEE Trans. Med. Imag., vol. 31, no. 12, pp. 2234-2240, Dec. 2012.

[41] A. Autret, J. Bert, O. Strauss, and D. Visvikis, "Fully 3D PET listmode reconstruction including an accurate detector modeling on GPU architecture," in Proc. 12th Int. Meet. Fully Three-Dimensional Image Reconstruct. Radiol. Nucl. Med., 2013, pp. 229-232.

[42] R. Prasad and H. Zaidi, "Scatter characterization and correction for simultaneous multiple small-animal PET imaging," Mol. Imag. Biol., vol. 16, pp. 199-209, 2014.

[43] A. M. Loening and S. S. Gambhir, "AMIDE: A free software tool for multimodality medical image analysis," Mol. Imag., vol. 2, no. 3, pp. 131-137, Jul. 2003.

[44] Philips, "Imalytics," [Online]. Available: http://www.philips.com/imalytics

[45] V. Tabacchini, V. Westerwoudt, G. Borghi, S. Seifert, and D. R. Schaart, "Probabilities of triggering and validation in a digital silicon photomultiplier," J. Instrum., vol. 9, no. 06, pp. P06016-P06016, June 2014.

[46] Am. College Radiol., Magnetic resonance imaging quality control manual, (ACR) 2004

[47] NEMA Standards MS 1-2008: Determination of Signal-to-Noise Ratio (SNR) in Diagnostic Magnetic Resonance Imaging, NEMA MS 1, Nat. Electr. Manufact. Assoc., 2008.

[48] NEMA Standards MS 3-2008: Determination of Image Uniformity in Diagnostig Magnetic Resonance Imaging, NEMA MS 3, Nat. Electr. Manufact. Assoc., 2008.

[49] NEMA Standards NU 4-2008: Performance Measurements of Small Animal Positron Emission Tomographs, NEMA NU 4, 2008.

[50] T. J. Havens, T. Duby, and J. Huang, "VRMS homogeneity definition: A proposal," in Proc. Int. Soc. Mag. Reson. Med., 2002, vol. 10, no. 4, p. 29501.

[51] J. Wehner and V. Schulz, "Hybrid PET/MRI Insert: B0 field optimization by applying active and passive shimming on PET detector level," IEEE Trans. Nucl. Sci., vol. 62, no. 3, pp. 644-649, Jun. 2015.

[52] P. Gebhardt et al., "RESCUE-Reduction of MR-SNR-degradation by using an MR-synchronous low interfering PET acquisition technique," IEEE Trans. Nucl. Sci., vol. 62, no. 3, pp. 634-643, Jun. 2015.

[53] H. F. Wehrl et al., "Assessment of MR compatibility of a PET insert developed for simultaneous multiparametric PET/MR imaging on an animal system operating at 7 T," Magn. Reson. Med., vol. 65, no. 1 , pp. 269-279, Jan. 2011

[54] S. Yamamoto et al., "Interference between PET and MRI sub-systems in a silicon-photomultiplier-based PET/MRI system," Phys. Med. Biol., vol. 56, no. 13, pp. 4147-4159, Jul. 2011.

[55] S. H. Maramraju et al., "Small animal simultaneous PET/MRI: Initial experiences in a 9.4 T microMRI," Phys. Med. Biol., vol. 56, no. 8, pp. 2459-2480, Apr. 2011.

[56] C. Catana et al., "Simultaneous acquisition of multislice PET and MR images: Initial results with a MR-compatible PET scanner," J. Nucl. Med., vol. 47, no. 12, pp. 1968-1976, Dec. 2006.

[57] W. W. Moses, "Time of flight in PET revisited," IEEE Trans. Nucl. Sci., vol. 50, no. 5, pp. 1325-1330, Oct. 2003.

[58] D. Schug et al., "ToF performance evaluation of a PET insert with digital silicon photomultiplier technology during MR operation," IEEE Trans. Nucl. Sci., vol. 62, no. 3, pp. 658-663, Jun. 2015.

[59] K. Büscher et al., "Isochronous assessment of cardiac metabolism and function in mice using hybrid PET/MRI," J. Nucl. Med., vol. 51, no. 8, pp. 1277-1284, Aug. 2010

[60] W. W. Lee et al., "PET/MRI of inflammation in myocardial infarction," J. Am. Coll. Cardiol., vol. 59, no. 2, pp. 153-163, Jan. 2012. 